\title{
Eclipsing binaries as IRAS sources
}

\author{
C. Friedemann ${ }^{1}$, J. Gürtler ${ }^{1}$ and M. Löwe ${ }^{1,2}$ \\ 1 Universitäts-Sternwarte, Schillergäßchen 2, D-07745 Jena, Germany \\ 2 Max-Planck-Gesellschaft, Arbeitsgruppe "Staub in Sternentstehungsgebieten", Schillergäßchen 2-3, D-07745 Jena, \\ Germany
}

Received July 31; accepted November 11, 1995

\begin{abstract}
In a systematic search we looked for coincidences in the positions of eclipsing binaries and IRAS point sources as evidence of a physical association. In a detailed discussion of the available optical and infrared data combined with model calculations, we show that 50-75\% of the coincidences between eclipsing binaries and IRAS sources are real. We discuss a subsample of 44 stars in some detail and surveyed the vicinity of them on POSS and SRC/ESO atlases for possible optical counterparts and present identification maps. In the cases of BS Sco and V 718 Sco the infrared radiation may come from an accretion disk.
\end{abstract}

Key words: binaries: eclipsing — circumstellar matter — stars: carbon — infrared: stars

\section{Introduction}

A good indicator of the presence of circumstellar matter is infrared excess emission. The IRAS satellite has provided us with an all-sky survey of infrared emission, resulting in the detection of about 250000 point sources. The infrared data allow to draw some conclusions on the physical nature and evolutionary state of the stars associated with the infrared point sources (see e.g. van der Veen \& Habing 1988; Walker et al. 1989; Smith et al. 1990). Therefore, a positive identification of a star or stellar group with infrared sources should be of great help.

Close binary stars are connected with various forms of circumstellar matter which cause different observable effects. A subgroup of binaries easily to be identified are the eclipsing variables. Our aim is to investigate the properties of circumstellar matter located in eclipsing variables by means of their infrared emission. Therefore, independently of the automatic search for associations of the infrared point sources with known objects we performed a systematic survey of eclipsing binaries with infrared point sources listed in IRAS point source catalogue. We will discuss the associations found in Sect. 2. A subsample of infrared sources for which at least three fluxes are measured by IRAS will be studied in more detail for other possible optical counterparts on the Palomar Observatory Sky Survey (POSS) and its extension to the southern sky (ESO/SRC Atlas).

Send offprint requests to: C. Friedemann

\section{Search strategy}

In a systematic searching procedure we looked for positional coincidences between eclipsing binaries and infrared point sources detected by the IRAS satellite as a first indication of a possible physical association. Following the strategy introduced with the possible associations listed in the IRAS Point Source Catalogue (Joint IRAS Science Working Group 1988), we restricted our search to angular separations $\leq 90^{\prime \prime}$. The positions of the eclipsing binaries are taken from the 4th edition of the General Catalogue of Variable Stars (GCVS, Kholopov et al. 1985). We found 233 IRAS point sources to fulfil this criterion. Hereafter we will refer to them as positionally associated sources or binaries. The majority of them involves Algol systems $(62.7 \%)$. This is in full accordance with the fact that most of the known eclipsing binaries have been classified as Algol systems. As the data in Table 1 show, the distribution of the associations over the various subtypes of eclipsing binaries follows the distribution of all known eclipsing binaries over the subtypes. No particular subtype is statistically significantly associated with IRAS sources, even if the $\beta$ Lyrae stars seems to be slightly more abundant among the infrared sources than among the total sample of eclipsing binaries.

The number of associations found considerably exceeds the number of positionally associations that may be expected by chance. Assuming the apparent distribution over the sky to be the same for both the eclipsing binaries and the IRAS point sources, we estimate that only 
Table 1. Statistics of the subtypes of eclipsing stars

\begin{tabular}{lrlrl}
\hline Subtype & \multicolumn{2}{l}{$\begin{array}{l}\text { All eclipsing stars } \\
\text { of GCVS }\end{array}$} & \multicolumn{2}{l}{$\begin{array}{l}\text { Eclipsing binaries } \\
\text { positionally associated } \\
\text { with IRAS sources }\end{array}$} \\
& $\begin{array}{l}\text { Total } \\
\text { number }\end{array}$ & $\begin{array}{l}\text { Relative } \\
\text { number }\end{array}$ & $\begin{array}{l}\text { Total } \\
\text { number }\end{array}$ & $\begin{array}{l}\text { Relative } \\
\text { number }\end{array}$ \\
\hline E & 817 & 0.158 & 36 & 0.155 \\
EA & 3022 & 0.584 & 146 & 0.627 \\
EB & 587 & 0.113 & 37 & 0.159 \\
EW & 562 & 0.108 & 13 & 0.056 \\
EX & 44 & 0.008 & 1 & 0.004 \\
\hline
\end{tabular}

60 associations should have been found. Consequently, the majority of the 233 associations detected must be real.

Figure 1 shows the number of positional associations as a function of the angular separation between the positions of the eclipsing binary and the IRAS source. If the coincidences were totally accidental, we would expect the number of associations to increase steadily with the separation. The distribution shown in Fig. 1 consists of two components. First, at small separations there is a distribution where the number of associations decreases with $D$. This distribution resembles a Gaussian curve and may originate from the errors in the IRAS positions. Thus, we expect that these close associations are mostly real coincidences. The second component of the distribution which dominates at separations $\geq 50^{\prime \prime}$ seems to be largely caused by accidental coincidences. From these distributions we estimate that our sample should contain about 150 real associations. This number agrees satisfactorily with the number of associations expected by chance estimated above. However, any particular coincidence cannot be excluded by statistical means alone. The reality of each association has to be proved or disproved by a detailed discussion of the infrared sources and their suggested counterparts..

In Fig. 2 we compare the brightness distributions for all eclipsing binaries after Herczeg (1982) and for those positionally associated with IRAS sources. A systematic trend is evident. The brighter stars $\left(V \lesssim 8^{\mathrm{m}}\right)$ are clearly more numerous among the possibly associated eclipsing binaries than among the total sample. We interprete this fact as an indication that the brighter stars are actually associated with the IRAS sources whereas only a small number of the fainter stars are true counterparts of infrared sources.

In Fig. 3 we plotted the $12-\mu$ m fluxes as observed by IRAS versus the visual magnitudes for all positionally associated binaries for which their spectral types are known. It can be seen that, within a spectral class, there is a correlation between both parameters for the brighter stars, indicating that the infrared emission for these stars is simply stellar radiation and the association of infrared source and binary is real. However, striking deviations from the mean

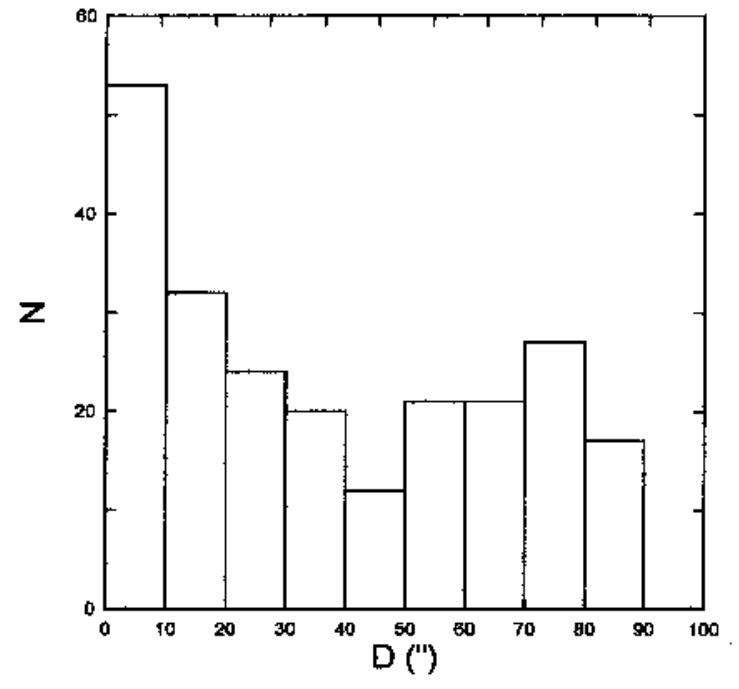

Fig. 1. Frequency distribution of eclipsing binaries positionally associated with IRAS point sources as function of angular separation $D$

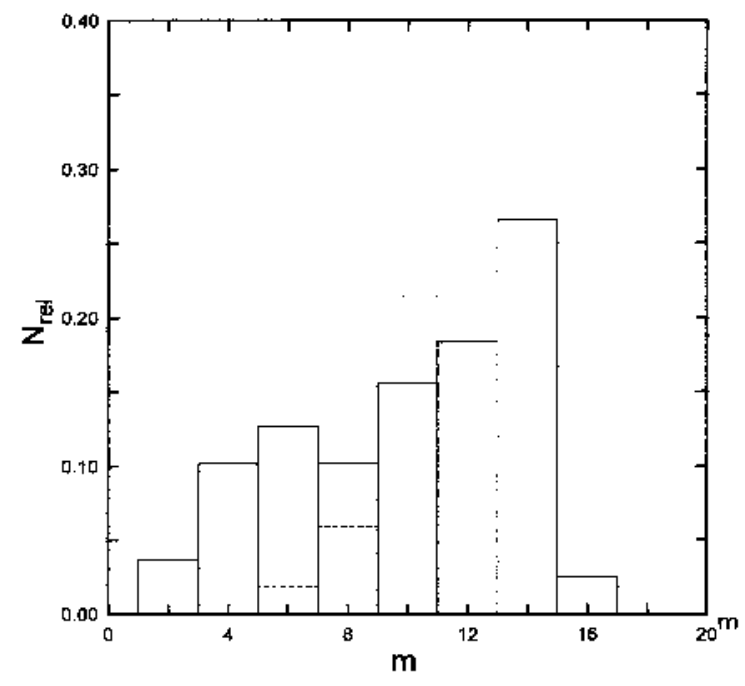

Fig. 2. Relative distribution of eclipsing binaries with their brightness. Broken line: distribution for all eclipsing binaries after Herczeg (1982); full line: distribution for eclipsing binaries possibly associated with IRAS sources

relationships occur. Infrared excesses, interstellar extinction or misidentifications may contibute to them.

Figures 4 and 5 show the two IRAS colour-colour diagrams for the infrared sources positionally associated with eclipsing binaries. All sources are plotted for which at least one colour index could be calculated. The colour indices are defined in the usual way as

$$
R\left(\lambda_{i} / \lambda_{i+1}\right)=\log \left(\frac{\lambda_{i} S\left(\lambda_{i+1}\right)}{\lambda_{i+1} S\left(\lambda_{i}\right)}\right)
$$




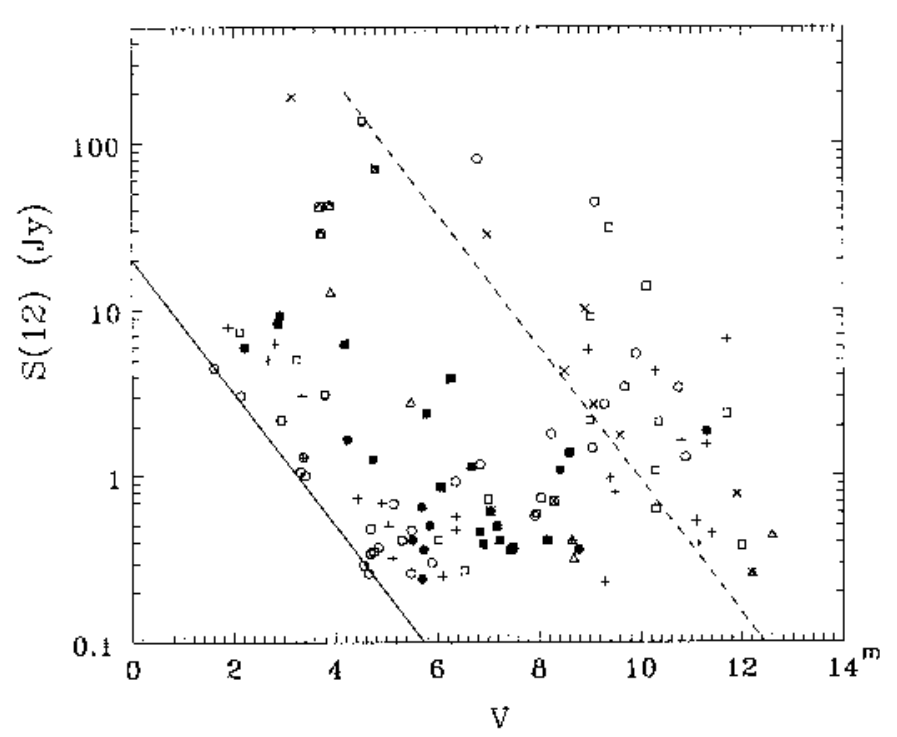

Fig. 3. The $12-\mu \mathrm{m}$ flux of the positionally associated IRAS point sources versus the visual magnitude of the eclipsing binaries with known spectral type. If the spectral type of both components is known, the star is plotted twice. The spectral types are coded as follows: open circles: O...B5, open squares: B6...B9, crosses: A, filled circles: F, filled squares: G, open triangles: K, diagonal crosses: M. The solid line gives the relation for unreddened early-type stars, the broken line is the mean relation for $\mathrm{M}$ stars

where the $\lambda_{i}$ is the effective wavelength of any of the four IRAS passbands and $S\left(\lambda_{i}\right)$ the flux in this passband. The distribution of the objects in the diagrams shows a large scatter, but some trends seem to be apparent even if we have to keep in mind that for many objects one colour index is an upper limit only and a number of additional objects could not be plotted at all. First, in the $R(12 / 25)-$ $R(25 / 60)$ diagram (Fig. 4) there seems to be a dividing line starting at $R(12 / 25) \approx-0.4, R(25 / 60) \approx-1.0$ and running to $R(12 / 25) \approx 1.0, R(25 / 60) \approx 1.0$ such that the upper left corner is free of objects. Second, there is a clear concentration of objects at the lower left corner of this diagram at $R(12 / 25) \approx-0.7, R(25 / 60) \approx-0.9$. In the $R(25 / 60)-R(60 / 100)$ diagram (Fig. 5$)$ the number of data points is significantly smaller. The main reason for this is that for the majority of the sources only upper limits for the $60-\mu \mathrm{m}$ and $100-\mu \mathrm{m}$ fluxes are available. Nevertheless, a similar dividing line as in Fig. 4 seems to exist.

The large scatter of the data points in Figs. 4 and 5 indicates that our sources do not form a homogeneous set of objects and that different processes are responsible for the infrared radiation. This can be further elucidated if we overlay the $R(12 / 25)-R(25 / 60)$ diagram (Fig. 4$)$ with the classification scheme proposed by van der Veen \& Habing (1988) (Fig. 6). From this diagram we conclude that most of the infrared sources possibly associated with eclipsing binaries belong to the classes I, II, IIIa, VIb, VII, and
VIII and should be normal stars and late-type stars with circumstellar dust shells of different evolutionary states.

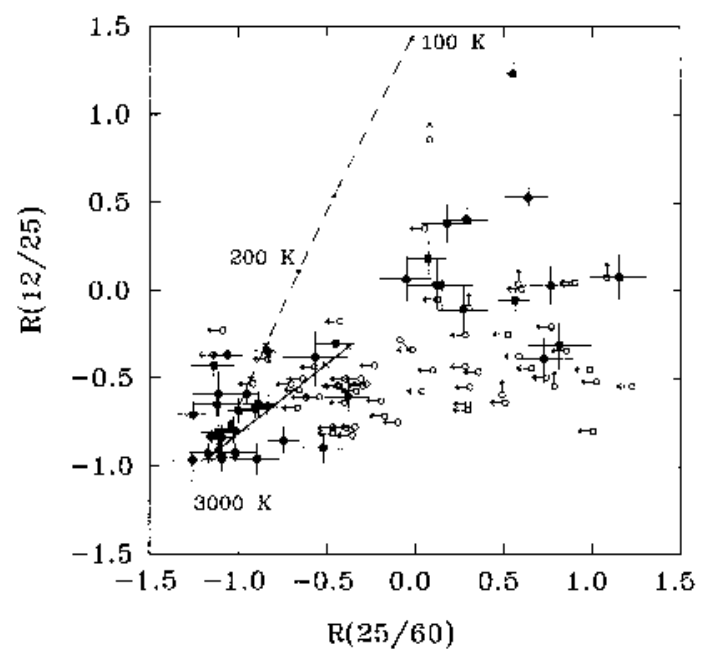

Fig. 4. $R(12 / 25)-R(25 / 60)$ diagram of the IRAS sources positionally associated with eclipsing binaries. The length of the crosses indicates the errors due to the uncertainty of the flux measurements. Arrows indicate colour indices computed from a data set containing an upper limit for one of the fluxes. The dashed curve marks the position of the black body line. The solid line is the position of sources with an accretion disk according to Smith et al. (1990)

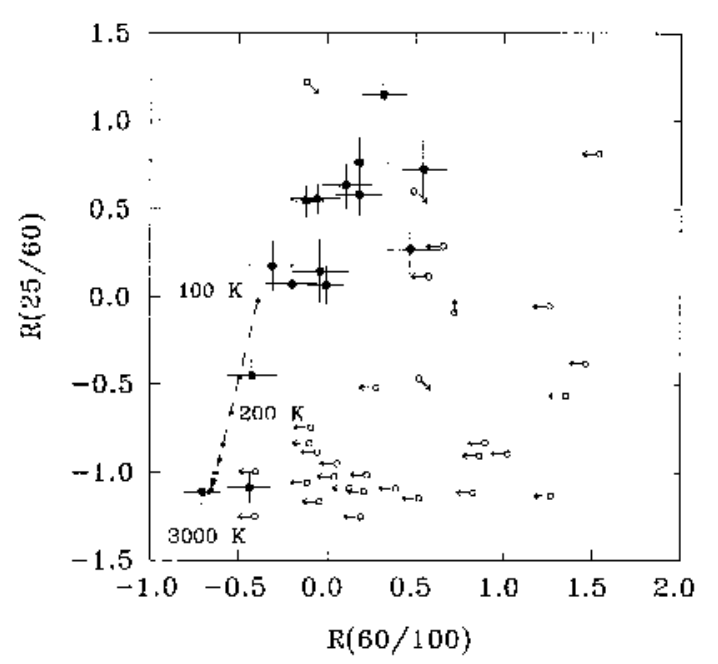

Fig. 5. $R(25 / 60)-R(60 / 100)$ diagram of the IRAS sources positionally associated with eclipsing binaries. The symbols have the same meaning as in Fig. 4

\section{Discussion of the restricted sample}

Most of the infrared sources positionally associated with eclipsing binaries are rather weak and part of the IRAS data are of poor quality. From our primary list of 233 
Table 2. List of eclipsing stars positionally associated with IRAS point sources: Restricted sample

\begin{tabular}{|c|c|c|c|c|c|c|c|c|}
\hline \multirow[t]{2}{*}{ Variable star } & \multirow{2}{*}{ Spectral type } & \multirow{2}{*}{ IRAS point source } & \multirow{2}{*}{$\begin{array}{c}\text { Separation } \\
\left({ }^{\prime \prime}\right)\end{array}$} & \multicolumn{4}{|c|}{ Infrared fluxes (Jy) } & \multirow{2}{*}{ Remarks } \\
\hline & & & & $12 \mu \mathrm{m}$ & $25 \mu \mathrm{m}$ & $60 \mu \mathrm{m}$ & $100 \mu \mathrm{m}$ & \\
\hline$\zeta$ And & K1II-III & $00446+2359$ & 15 & 12.91 & 2.92 & $0.39:$ & $<1.00$ & A \\
\hline$\delta$ Cas & A5V & $01225+5958$ & 9 & 4.96 & 1.14 & $0.35:$ & $<6.09$ & $\mathrm{~A}$ \\
\hline V 436 Per & $\mathrm{B} 1.5 \mathrm{~V}$ & $01487+5453$ & 24 & 0.26 : & 0.82 & 2.30 & 3.74: & $\mathrm{C}$ \\
\hline V 559 Cas & B9V & $02219+6119$ & 11 & $0.72:$ & 1.53 & $<14.66$ & 74.99: & $\mathrm{C}$ \\
\hline BM Eri & M6III & $04111-1030$ & 1 & 28.24 & 11.48 & 1.54 & $<1.00$ & A, LRS \\
\hline$\gamma$ Dor & F0V-F5V & $04147-5136$ & 1 & 1.66 & 0.49 & 0.32 : & $<1.00$ & A \\
\hline$\epsilon$ Aur & A8Ia-F2epIa+BV & $04583+4345$ & 4 & 9.25 & 2.79 & 0.54 & $<2.18$ & $\mathrm{~A}$ \\
\hline$\zeta$ Aur & $\mathrm{K} 5 \mathrm{II}+\mathrm{B} 7 \mathrm{~V}$ & $04589+4100$ & 4 & 41.86 & 9.89 & 1.93 & 1.17: & A, LRS \\
\hline VV Ori & $\mathrm{B} 1 \mathrm{~V}+\mathrm{B} 7 \mathrm{~V}$ & $05309-0111$ & 16 & 0.41 & 2.06 & 7.42 & 6.06 & $\mathrm{C}$ \\
\hline$\zeta \mathrm{Tau}$ & B1eIV+G8III: & $05346+2106$ & 10 & 8.30 & 3.87 & 1.21 & $<1.76$ & A, LRS \\
\hline EF Ori & & $06076+1251$ & 27 & 0.45 & 0.46 : & 7.20 & $<411.94$ & $\mathrm{~B}, \mathrm{C}$ \\
\hline$\eta$ Gem & M3IIIab & $06118+2231$ & 4 & 190.10 & 47.06 & 7.66 & $<11.48$ & A, LRS \\
\hline MQ Pup & B7V & $07295-3753$ & 30 & 9.03 & 4.20 & $0.77:$ & $<8.53$ & $\mathrm{~A}$ \\
\hline YY Gem & $\mathrm{M} 1 \mathrm{eV}+\mathrm{M} 1 \mathrm{eV}$ & $07314+3159$ & 21 & 10.14 & 2.31 & 0.45 & $<1.00$ & A, LRS \\
\hline FX Vel & $\mathrm{Be}$ & $08307-3748$ & 17 & 3.42 & 3.20 & 1.13 & $<14.61$ & A \\
\hline GP Vel & B0.5eIa Q & $09002-4020$ & 49 & $<0.57$ & 8.55 & 24.37 & 25.44: & $\mathrm{C}$ \\
\hline DQ Car & A0 & $10201-5700$ & 74 & 0.53 & $0.45:$ & 5.75: & 33.28: & $\mathrm{B}$ \\
\hline V438 Cen & & $11156-3754$ & 9 & 7.29 & 2.43 & 0.55 & $<1.00$ & A \\
\hline SY Cru & & $12043-6225$ & 52 & 29.30 & 22.73 & 4.01: & $<122.95$ & A, LRS \\
\hline BB Cru & & $12091-6129$ & 12 & 2.11 & 74.78 & 633.35 & 794.73 & $\mathrm{C}$ \\
\hline AA Cru & & $12146-6212$ & 84 & 4.12 & 29.03 & 301.08 & 637.81: & $\mathrm{C}$ \\
\hline BU Cru & $\mathrm{B} 1.5 \mathrm{Ib}$ & $12506-3753$ & 40 & 80.71 & 43.18 & 11.63 & $<21.90$ & A, LRS \\
\hline NX Cen & & $13211-6227$ & 52 & 6.45 & 10.48 & 46.72: & 228.94: & C \\
\hline AU Cir & & $14394-6630$ & 69 & 0.22 : & $<0.24$ & 0.47 & 4.06 & $\mathrm{~B}$ \\
\hline AT Nor & & $15541-5724$ & 58 & 4.68 & 1.50 & $<1.23$ & 6.71: & $\mathrm{B}$ \\
\hline V 718 Sco & A8III-IV & $16102-2221$ & 12 & 5.68 & 5.89 & 5.01 & 3.14 & $\mathrm{~B}$ \\
\hline AF Ara & & $16331-5537$ & 77 & 9.83 & 4.30 & 1.29 & $<15.52$ & $\mathrm{~A}$ \\
\hline$\epsilon \mathrm{UMi}$ & G5III & $16510+8207$ & 0 & 6.22 & 1.55 & 0.36 : & $<1.00$ & $\mathrm{~A}$ \\
\hline V 463 Sco & & $17079-3243$ & 80 & 146.92 & 62.78 & 15.20 & $<10.02$ & A, LRS \\
\hline V 499 Sco & $\mathrm{B} 1 \mathrm{~V}$ & $17256-3258$ & 58 & 1.79: & 4.33 & 9.22: & $<276.83$ & $\mathrm{C}$ \\
\hline BS Sco & F8 & $17518-3138$ & 36 & 2.35: & 2.04 & 1.33: & $<49.29$ & A \\
\hline V 792 Sgr & & $18000-2252$ & 20 & 1.77 & 9.26 & 42.91 & $<319.37$ & $\mathrm{C}$ \\
\hline W Sct & $\mathrm{B} 3 \mathrm{n}+\mathrm{B} 0$ & $18218-1340$ & 65 & 5.38 & 12.14 & 38.20: & $<234.96$ & $\mathrm{C}$ \\
\hline RY Sct & O9.7Ib pec & $18227-1243$ & 13 & 44.28 & 22.84 & 22.80 : & $<1094.81$ & $\mathrm{~B}$ \\
\hline$\beta \mathrm{Lyr}$ & B8II-IIIep & $18482+3318$ & 21 & 4.99 & 2.27 & 0.80 & $<1.05$ & $\mathrm{~A}$ \\
\hline TY CrA & $\mathrm{B} 9 \mathrm{eV}$ & $18583-3657$ & 10 & 30.99 & 57.01 & 496.67 & 719.06 & $\mathrm{C}$ \\
\hline V 1062 Sgr & & $18594-2042$ & 75 & 4.85 & 2.58 & $0.48:$ & $<1.29$ & A \\
\hline$v \mathrm{Sgr}$ & B8Ip+O9V & $19188-1603$ & 3 & 136.63 & 44.19 & 8.15 & 2.67 & A, LRS \\
\hline HP Lyr & A6 & $19199+3950$ & 3 & 4.23 & 3.78 & 0.79 & $<1.24$ & $\mathrm{~A}$ \\
\hline$\sigma$ Aql & $\mathrm{B} 3 \mathrm{~V}+\mathrm{B} 3 \mathrm{~V}$ & $19366+0517$ & 30 & 0.68 : & 1.53 & 5.11 & 7.71: & $\mathrm{C}$ \\
\hline EV Vul & B1V: & $19495+2345$ & 15 & $<0.25$ & 0.57 & 5.22: & 13.10 & $\mathrm{C}$ \\
\hline V 501 Cyg & & $20241+3357$ & 71 & 0.32 & 0.19 : & $<7.62$ & 9.61: & $\mathrm{B}, \mathrm{C}$ \\
\hline VV Cep & M2epIa-Iab+B8:ev & $21552+6323$ & 8 & 70.28 & 21.54 & 3.66 & $<20.08$ & A, LRS \\
\hline PS Cep & B6Vne & $22457+5751$ & 81 & 13.82 & 30.80 & 431.31 & 1088.05: & $\mathrm{C}$ \\
\hline
\end{tabular}

eclipsing variables associated with IRAS point sources, we, therefore, have built a subsample of objects for which at least 3 of the infrared fluxes measured by IRAS have the quality 1 or 2 . We imposed this restriction to have reasonable information about the spectral energy distribution. Altogether 44 objects fulfil this condition. In Table 2 we have summarized relevant data for them. Unfortunately, there are only 10 objects for which IRAS low resolution spectra are available (Fig. 8).

Additional information on the nature of the infrared radiation can be obtained from the spectral energy distribution (SED). (It must be noted that the IRAS sources positionally associated with eclipsing binaries for convenience are labelled with their names in Fig. 7 and the text). In Fig. 7 we show the SEDs for all objects of our restricted sample. As the shapes of the SEDs are quite different, we roughly form three groups. This classification is given in the last column of Table 2 . It is ambiguous in a few cases.

Group A: The SEDs are characterized by the IRAS fluxes decreasing with increasing wavelength roughly $\propto$ $\lambda^{-1}$ or steeper. For a blackbody of stellar temperature we expect a SED $\propto \lambda^{-2}$ in the infrared. The SEDs of the stars of this group are in general flatter and can be understood as emission from rather hot dust or seldom as stellar emission.

Group B comprises objects whose SED is quite flat or shows a minimum. Such SEDs are difficult to interprete. 


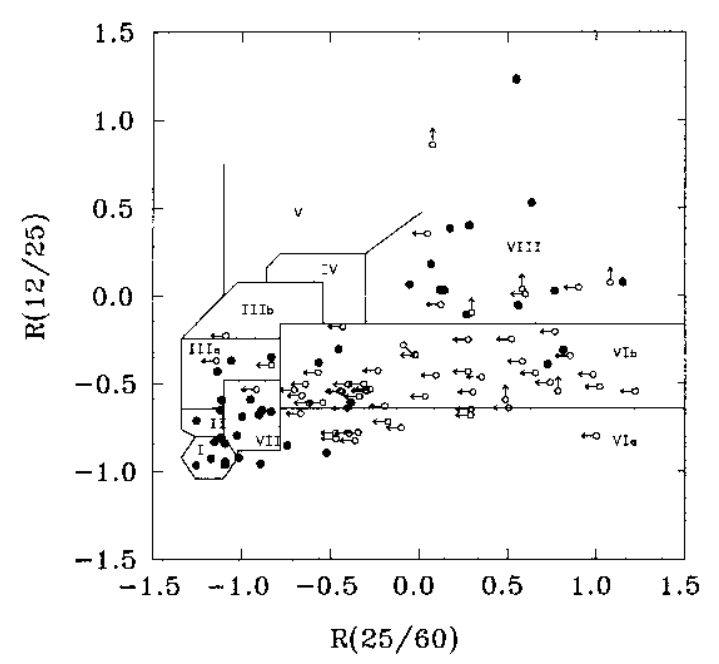

Fig. 6. $R(12 / 25)-R(25 / 60)$ diagram of the IRAS point sources positionally associated with eclipsing binaries overlayed with the classification grid of van der Veen \& Habing (1988). The symbols have the same meaning as in Fig. 4

Free-free emission, warm dust shells, and disk-like configurations have to be considered.

Group C: The SED is characterized by a steep increase of the infrared emission with wavelength (roughly $\propto \lambda$ ). The SEDs are often peaking at $100 \mu \mathrm{m}$ or beyond and can be explained as radiation from cold dust. Very young objects embedded in molecular clouds but also evolved objects with very extended shells possess such SEDs.

Our grouping is also reflected in the distribution of the sources in the colour-colour diagram (Fig. 4). Obviously, the members of Group A flock together close to the blackbody line. The range of temperatures indicated for the Group A objects is typical of the coolest stars, while the temperatures for the Group B objects are lower than the temperatures of the coolest stars known. Hence, a stellar origin of the radiation is excluded. In Fig. 4 we also indicated the location of objects with optically thick accretion disks after Smith et al. (1990). Two of our sources (BS Sco and V $718 \mathrm{Sco}$ ) could be interpreted in this way.

The members of Group $C$ populate a larger area of the $R(12 / 25)-R(25 / 60)$ diagram than the other two groups. This area is the typical site of objects with extended dust envelopes of various sizes and optical thicknesses (see, e.g., Gürtler et al. 1991).

Comparing the positions of our objects in the colourcolour diagram with the classification by van der Veen \& Habing (1988), our objects belong to a number of their classes. For instance, our group A is comparable to their classes I ("oxygen-rich non-variable stars without circumstellar shells"), II ("variable stars with 'young'O-rich circumstellar shells"), VII ("variable stars with more evolved C-rich circumstellar shells") and IIIa ("variable stars with more evolved O-rich circumstellar shells"). Our group B corresponds to the van der Veen \& Habing classes VIa ("non-variable stars with relatively cold dust at large distances; a significant part of the stars is carbon-rich") and VIb ("variable stars with relatively hot dust close to the star and relatively cold dust at large distances; some of the objects have proven to be O-rich"). The correspondence between our group C and any of van der Veen \& Habing's classes is not as straightforward. Part of the objects may be classified as VIb, but most of them are located in the region VIII ("different sort of objects, but mostly associated with very cold dust").

From their distribution in the colour-colour diagrams (Figs. 4 and 5) we have to conclude that the eclipsing binaries positionally associated with IRAS sources comprise a large variety of physically different infrared emitters. Keeping in mind that the colour index of a number of our objects is an upper limit only (points marked with arrows in Figs. 4 and 5), our objects concentrate in the lower left corner of Fig. 4. This concentration reflects the distribution found by van der Veen \& Habing (1988) for all IRAS sources (cf. their Fig. 5a). It is interesting to note that many bright stars of our sample populate the region of the van der Veen \& Habing class I, which means that we observe the radiation of normal stars in the infrared (Fig. 6).

The scrutiny of each object is still more decisive for the objects situated in the van der Veen \& Habing regions VIII and VIb (Fig. 6). On the other hand, model calculations for dust shells around hot stars show that the entire area of regions VIb and VIII can be covered with models with plausible parameters (Gürtler et al. 1991). In this context it is interesting to note that as far as spectral types are known, the stars are of early type, mostly B. In the following section we will study in more detail how trustworthy the associations between eclipsing binaries and IRAS sources found on the basis of positional agreement really are.

\section{Discussion of individual objects}

The decision on a possible physical association of an IRAS source with an eclipsing binary can be based only on a detailed discussion of all available information on the objects. In the following we will summarize the information presently available in an attempt to reach a conclusion on whether the association of the IRAS source with the eclipsing binary is probable or not. The information includes the visual brightness, spectral type, and interstellar extinction of the variable and the spectral energy distribution (SED) as well as the low resolution spectra (LRS) if known of the IRAS sources (Figs. 7 and 8). In addition, we searched the vicinity of the binaries for red objects on the POSS and ESO/SRC atlases. Figure 9 presents maps 


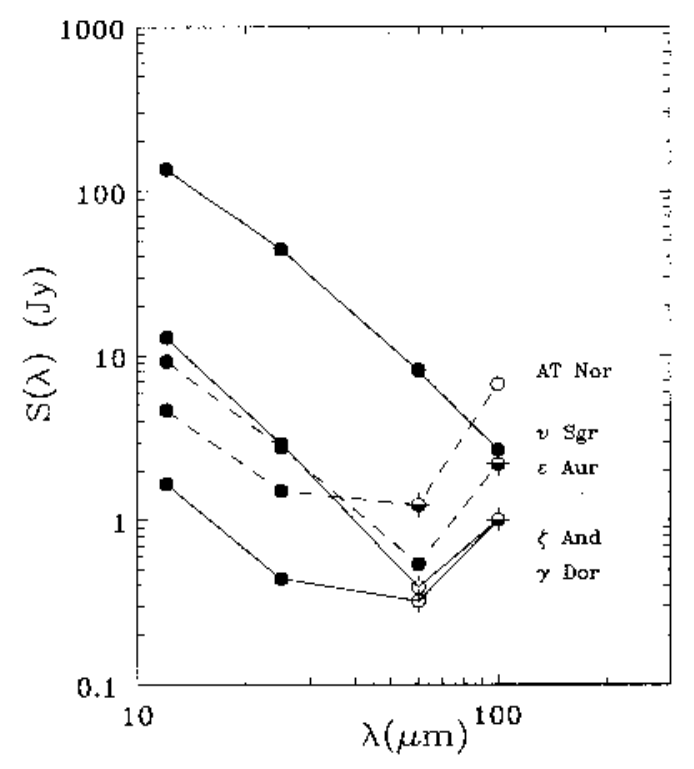

a)

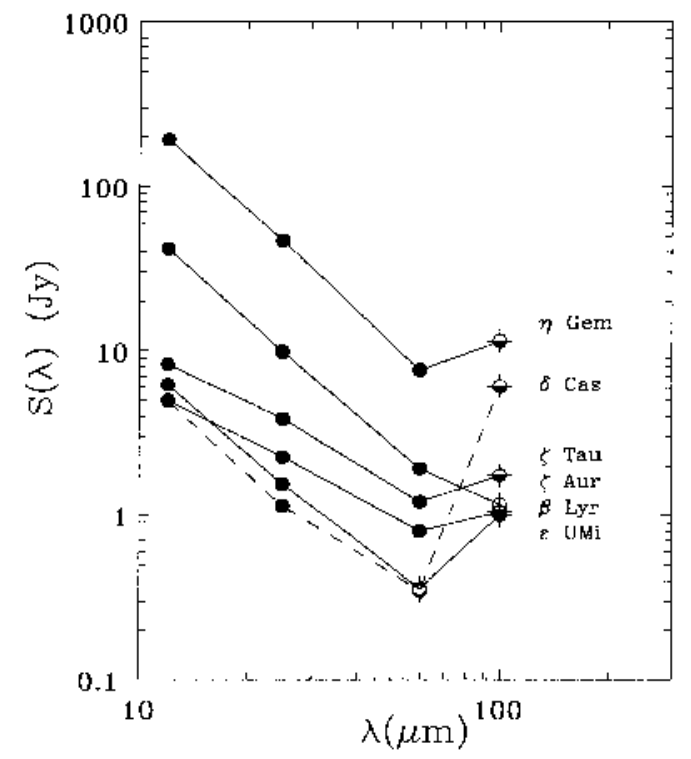

b)



c)

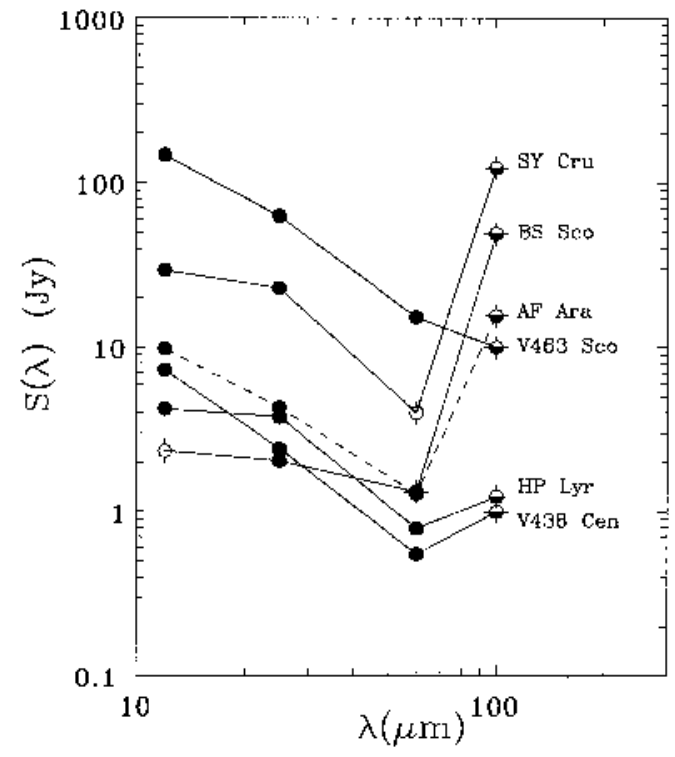

d)

Fig. 7. Spectral energy distribution of the objects of the restricted sample. For convenience the IRAS sources seemingly associated with eclipsing binaries are labelled with their names. The symbols used have the following meaning: points: certain values. open circles: uncertain values, half-filled circles: upper limits 


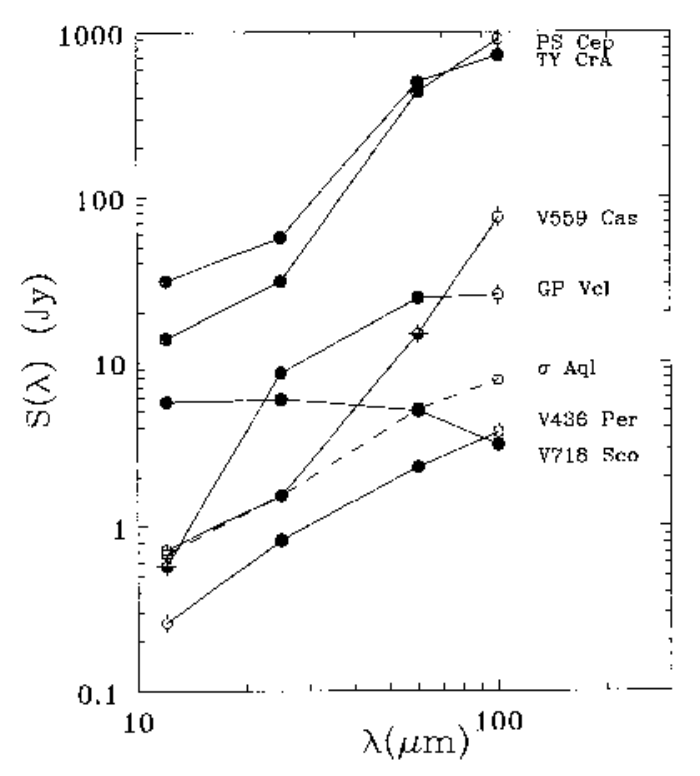

e)

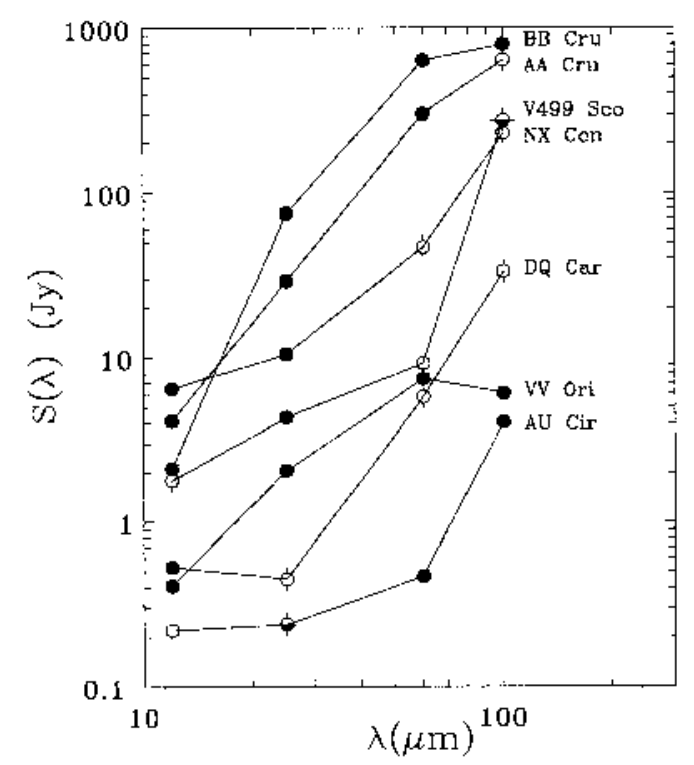

f)

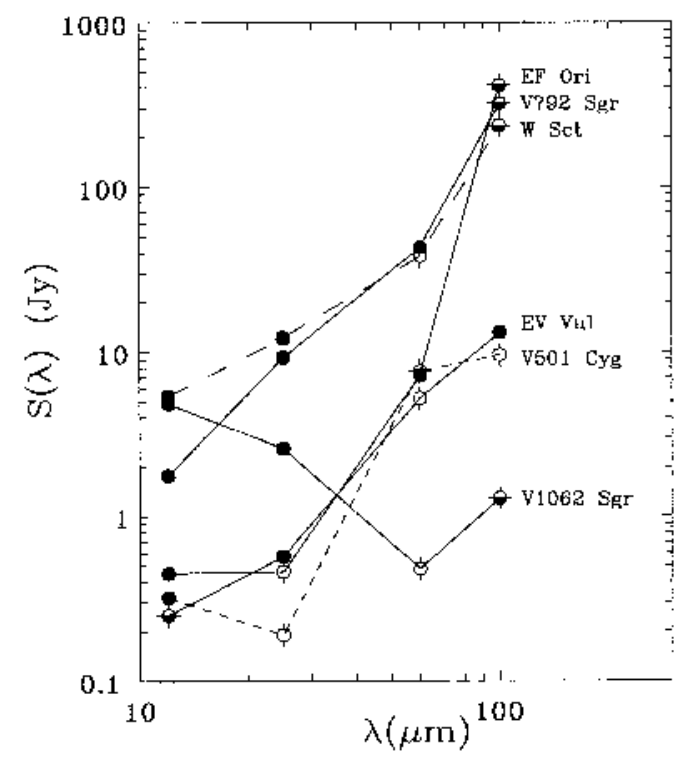

g)

Fig. 7. continued

for the identification of the eclipsing variables, red objects, and the IRAS positions. ${ }^{1}$

\footnotetext{
${ }^{1}$ The maps are based on the Digitized Sky Survey, produced at the Space Telescope Science Institute under U.S. Government grant NAC W-2166. Original plate material is copyright (C) the Royal Observatory Edinburgh and the Anglo-Australian Observatory.
}

The infrared radiation observed by IRAS is fully compatible with originating from the star in the following cases: $\zeta$ And, $\delta$ Cas, $\gamma$ Dor, $\epsilon$ Aur, $\zeta$ Aur, $\eta$ Gem, $\epsilon U M i$, and $V V$ Cep.

$V 436$ Perse : The infrared emission increases roughly $\propto \lambda$ indicating the presence of warm circumstellar dust. In this respect the binary resembles VV Ori and W Sct. 

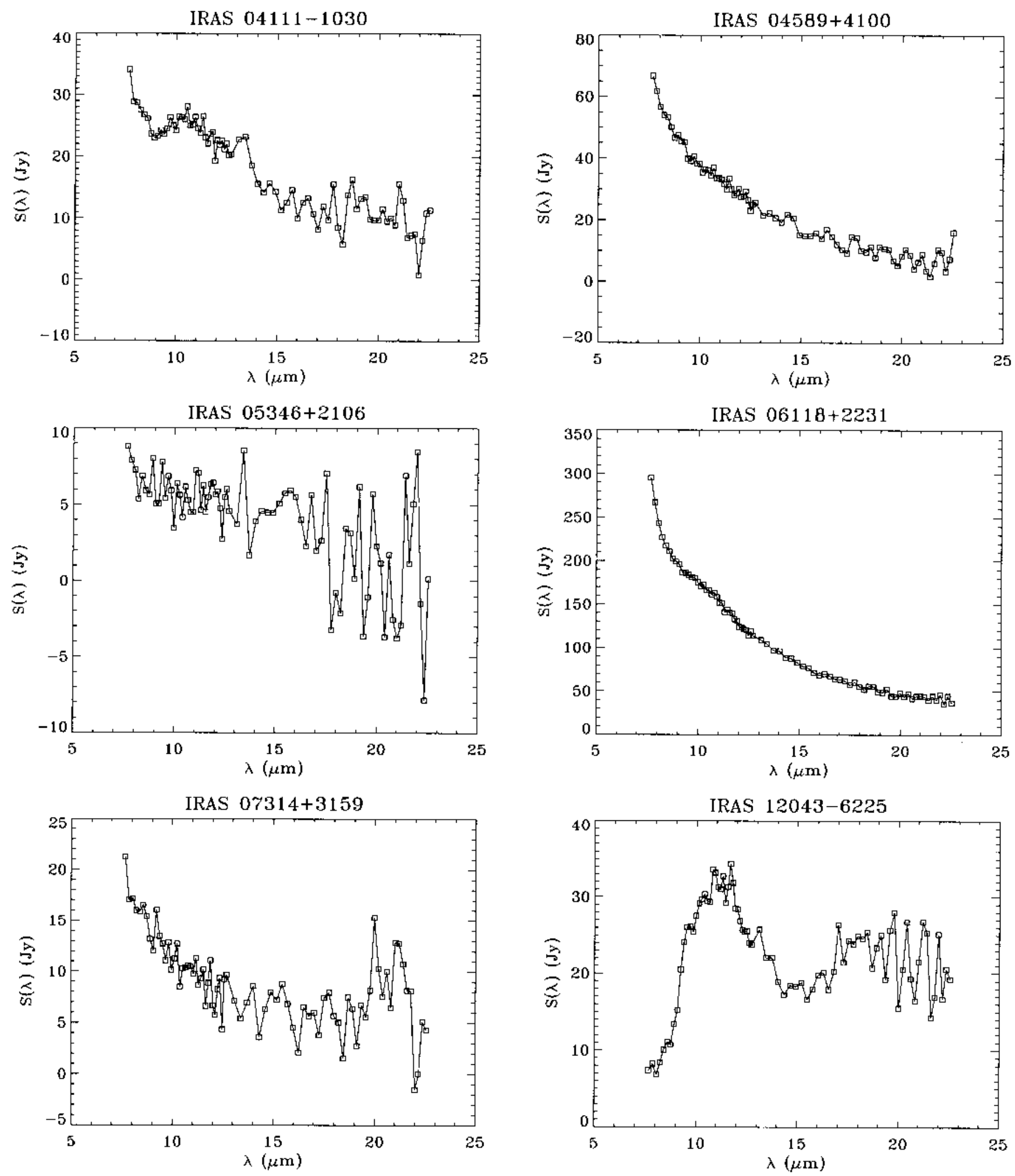

Fig. 8. IRAS low resolution spectra of point sources positionally associated with eclipsing binaries 

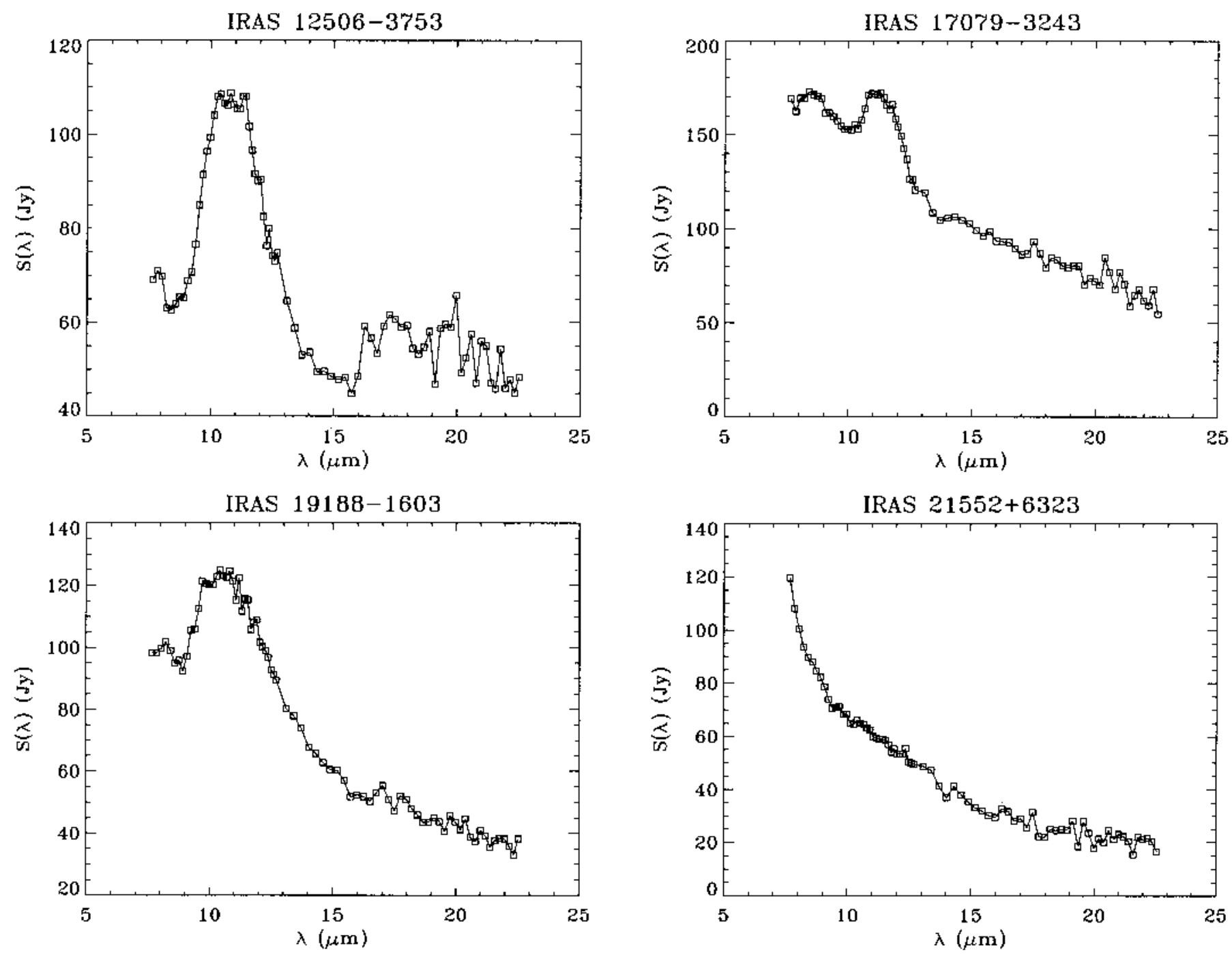

Fig. 8. continued

$V 559$ Cassiopeiae: The emission at $12 \mu \mathrm{m}$ is about 1 order of magnitude larger than one may expect from the visual magnitude. Furthermore, the infrared emission peaks beyond $100 \mu \mathrm{m}$. Therefore, we feel the identification of the binary with the IRAS source not convincing. A search on the POSS prints for a more probable candidate is excluded by the large stellar image.

BM Eridani: The IRAS LRS spectrum (Fig. 8) shows the $10-\mu \mathrm{m}$ silicate band in emission. The presence of this band is compatible with the spectral type of the binary. Therefore, star and IRAS source are identical.

$V V$ Orionis: From the IRAS fluxes we infer that this object belongs to group C, i.e. the infrared SED seems to be compatible with thermal emission from cool circumstellar dust. The existing NIR data (Gezari et al. 1993) indicate that the NIR radiation is emitted from the hot component (spectral type B1 V) of the binary. The ratio of the IRAS fluxes to the NIR fluxes is consistent with VV Ori being the counterpart of the IRAS source.

$\zeta$ Tauri: The location in the $S(12)-V$ diagram (Fig. 3 ) shows a weak infrared excess. The presence of the circumstellar emission is also indicated by the LRS spectrum (Fig. 8) and the SED. The spectrum is very noisy so that the origin of the excess radiation is not clear.

EF Orionis: This object belongs to Group C because the infrared emission peaks at $60 \mu \mathrm{m}$ or beyond according to the IRAS data. Little information on the variable is available. An identification of the IRAS source with the variable is not unambiguous. Inspection of the POSS prints revealed a red star at a distance of $\sim 1^{\prime}$ from the variable.

MQ Puppis: The IRAS fluxes decrease $\propto \lambda^{-1}$, which is indicative of circumstellar emission. Spectral type and visual magnitude are not compatible with the large infrared 

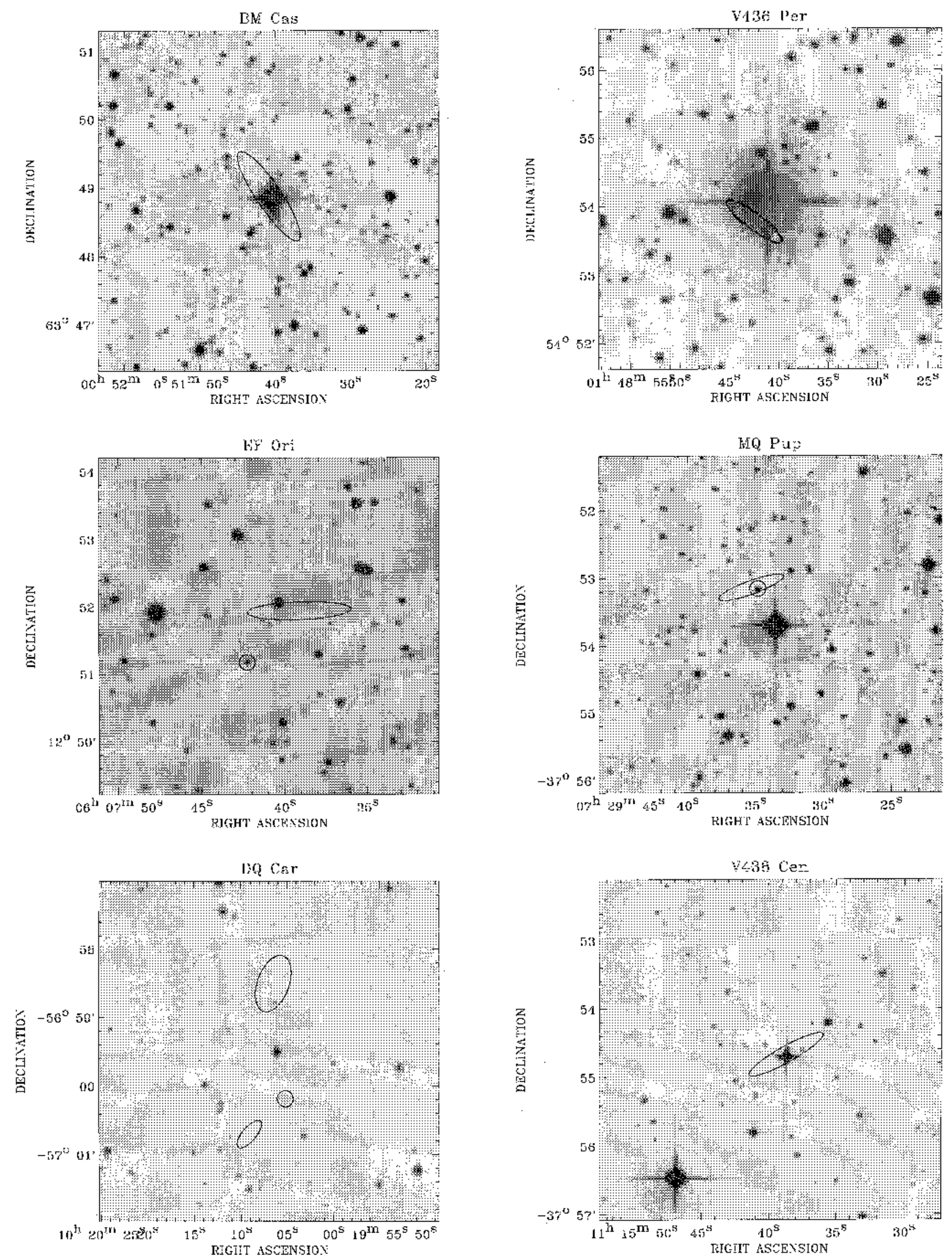

Fig. 9. Finding maps (based on the Digitized Sky Survey) for the eclipsing binaries and the IRAS point sources possibly associated with them. The variables are normally at the centres of the fields, otherwise they are marked by squares. The position of the IRAS source is marked by an ellipse, where the length of the axes give the $1 \sigma$ uncertainty of the position. Red objects found from inspection of the POSS and ESO-SRC atlases and suggested as possible counterparts of the IRAS source 

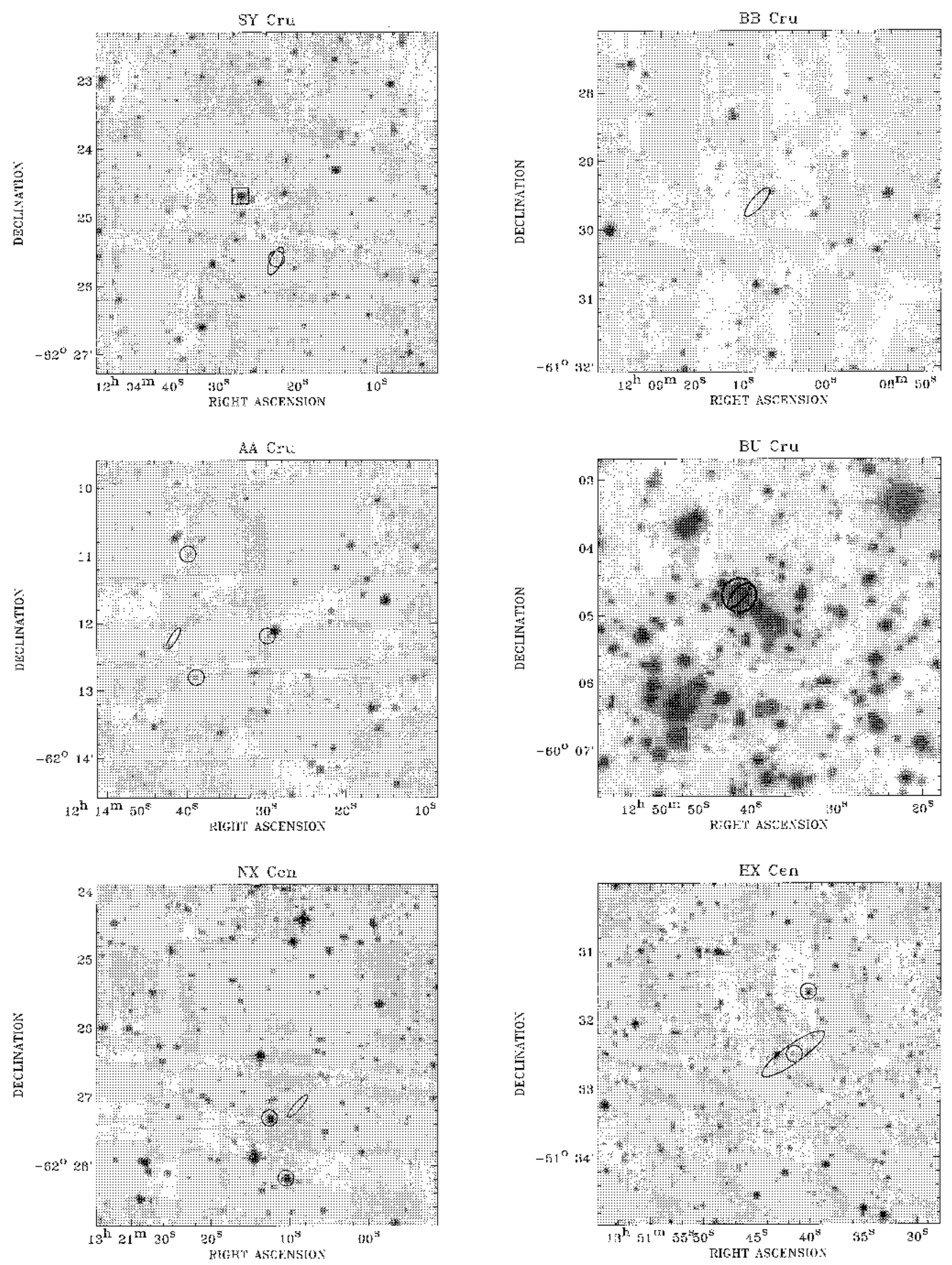

Fig. 9. continued 

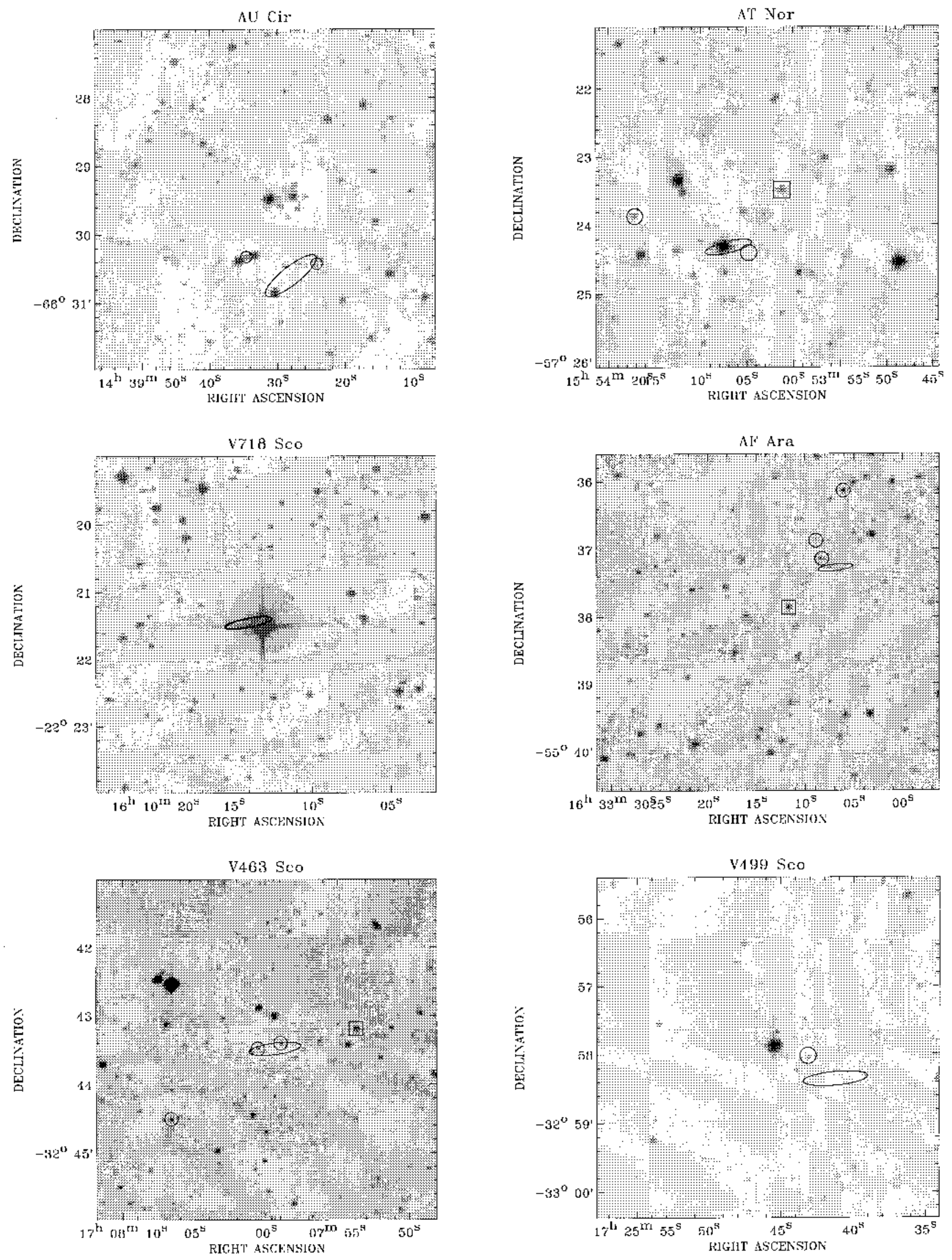

Fig. 9. continued 

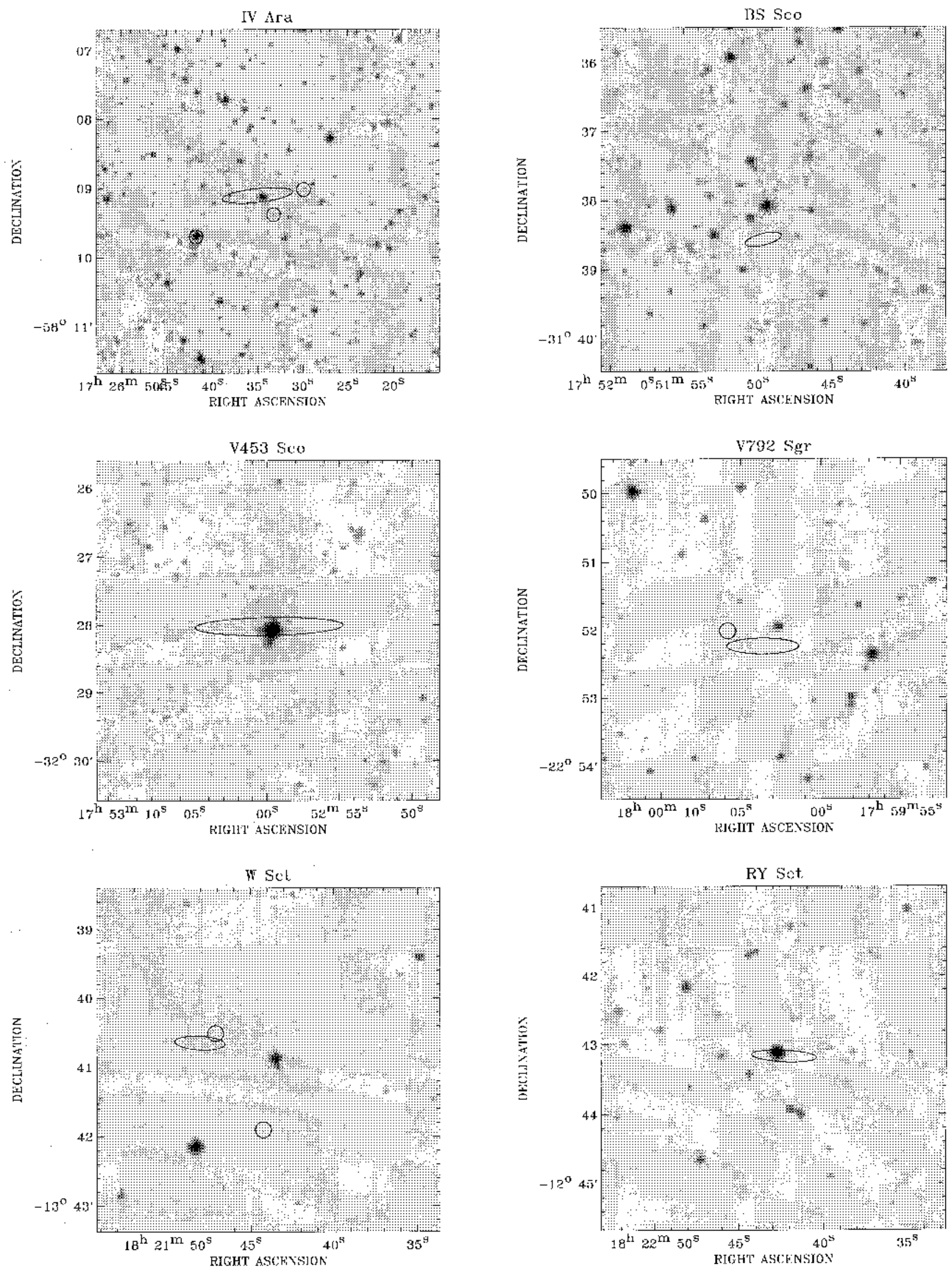

Fig. 9. continued 



Fig. 9. continued 

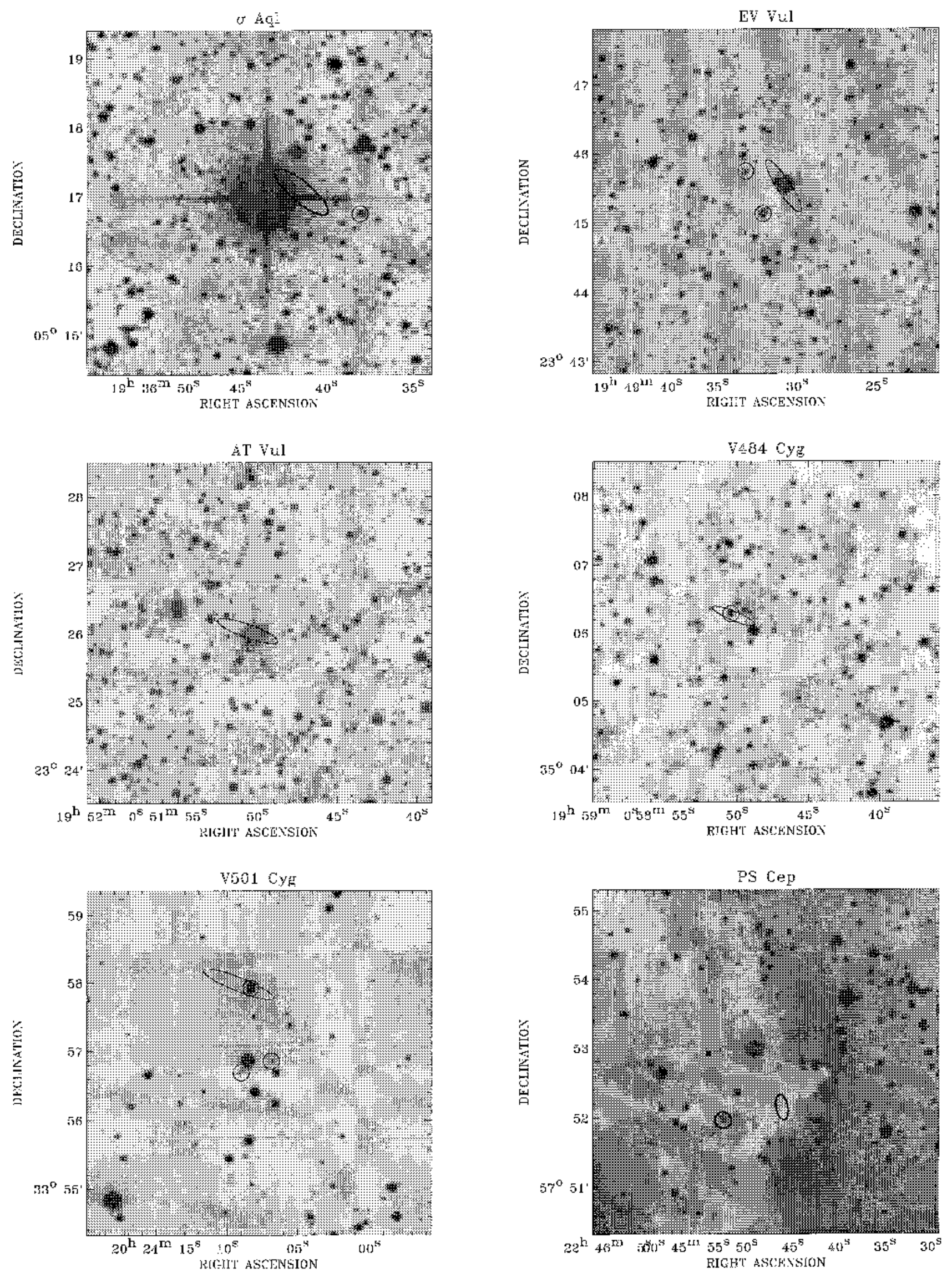

Fig. 9. continued 
flux at $12 \mu \mathrm{m}$. Therefore, we conclude that MQ Pup is not identical with IRAS $07295-3753$.

Inspection of the SRC/ESO Atlas revealed a red star northeast of the variable and coinciding well with the IRAS position. Therefore, we suggest this red star to be the optical counterpart of IRAS 07295-3753.

YY Geminorum: The eclipsing binary is a member of the $\alpha$ Geminorum system. Both the optical brightness and the smooth LRS spectrum (Fig. 8) points to $\alpha$ Gem AB $=$ Castor $\mathrm{AB}(V=1.97)$ as the counterpart of IRAS $07314+3159$.

FX Velorum: If the star is identical with the IRAS source, it belongs to our objects with the largest infrared excesses. The SED is quite flat in the infrared and may point to free-free emission as origin of the infrared radiation. Dong \& Hu (1991) suggested the identification of the IRAS source with the Be star CD -37 4833, which is identical to FX Vel.

No other promising candidate could be found from the inspection of the ESO-SRC Atlas.

GP Velorum: This star is identical with the X-ray source Vela X-1. Its spectral type points to the presence of a gaseous shell. The coincidence with IRAS 09002-4020 was also noted by Smith et al. (1990). The IRAS fluxes reveals the presence of cold dust. The binary may be surrounded by a fossil dust shell.

$D Q$ Carinae: Two IRAS sources are positionally associated with the variable. For IRAS 10201-5658 only the $25-\mu \mathrm{m}$ flux was measured by the satellite. Therefore, we exclude this source from the further discussion. The SED of IRAS 10201-5700 attaches the object to our group C, but the observations are uncertain. The infrared emission peaks beyond $100 \mu \mathrm{m}$ and points to cold circumstellar dust.

Inspection of the ESO/SRC Atlas revealed a red object about $45^{\prime \prime}$ south of the variable. Since the IRAS position agrees better with the position of the red star than with that of the eclipsing variable, we suggest the red star to be the counterpart of the IRAS source. According to the classification by van der Veen \& Habing (1988), the IRAS source belongs to their class VIb and is, therefore, probably an O-rich variable.

$V 438$ Centauri: The IRAS fluxes vary $\propto \lambda^{-1.6}$. The infrared source belongs to the van der Veen \& Habing class VII, and the optical counterpart is expected to be a carbon star and very red object. The spectral type of the eclipsing binary is not known. From the inspection of the ESO/SRC Atlas we conclude that the variable is blue rather than red. Moreover, there is no plausible assumption of the effective temperature of the star that makes the visual magnitude of the variable compatible with the IRAS data. The SED in the infrared is very similar to that of IRAS $17080-3243$ (V $463 \mathrm{Sco}$ ), which is a star with a C-rich shell according to its IRAS low resolution spectrum (Fig. 8). Therefore, we feel it is very unlikely that V 438 Cen should be identified with IRAS 11156-3754. Any red object in the immediate vicinity of the binary could not be found. Considering its large brightness and the good positional coincidence of the stellar and IRAS positions, we cannot exclude that a red object is hidden in the stellar image.

SY Crucis: The SED in the infrared as revealed by the IRAS fluxes shows a slight decrease toward longer wavelengths, indicating emission by hot dust. The existing low resolution spectrum (Fig. 8) displays the 10- and 18- $\mu \mathrm{m}$ silicate features in emission. Therefore, the optical counterpart of the IRAS source is very probably an evolved star with an optically thin O-rich shell.

The spectral type of SY Cru is not known, but the star is too faint to be the counterpart of the infrared source. Inspection of the SRC-ESO Atlas revealed a red object $\sim 50^{\prime \prime}$ south of the eclipsing star. Caldwell et al. (1991) identified this star as a long-period variable. Therefore, it seems proved that this red star and not SY Cru is the counterpart of IRAS 12043-6225.

BB Crucis: The IRAS fluxes show a steep increase up to $100 \mu \mathrm{m}$, indicating the emission by cold dust. Two types of dust shells are compatible with the infrared data. First, the shell may be optically thick in the visual and nearinfrared parts of the spectrum. Then we would expect an extremely weak or even invisible optical counterpart. Second, a very extended, but optically thin shell can also produce the observed SED. Then, the brightness of the associated star is roughly estimated at $16^{\mathrm{m}}$. Inspection of the ESO/SRC Atlas showed no promising candidate for a counterpart of the infrared source. We conclude, therefore, that the infrared source is an object with an optically thick dust shell.

AA Crucis: The infrared properties of the associated IRAS source are very similar to that of BB Cru. The inspection of the ESO/SRC Atlas showed a weak red object in the immediate vicinity of AA Cru which we suggest to be the possible optical counterpart of the IRAS source.

$B U$ Crucis: The eclipsing binary is situated in the open cluster NGC 4755 ( $=\kappa$ Cru). Its spectral type and visual magnitude contradicts its identification with the IRAS source. The LRS spectrum (Fig. 8) shows the silicate bands in emission. The IRAS fluxes points to the presence of rather cool dust.

Inspection of the identification map for NGC 4755 given by Arp \& van Sant (1958) showed that their star $\mathrm{D}$ is close to the IRAS position. Feast (1963) gives M2Ib as spectral type and $V=7^{\mathrm{m}} 58$ for this star. It is certain that this star and not BU Cru is the counterpart of IRAS 12506-6004.

NX Centauri: The infrared source belongs to our group C. The infrared radiation increases roughly as $\lambda^{2}$ and peaks beyond $100 \mu \mathrm{m}$. Hence, the object is associated with very cold dust. In the colour-colour diagram (see Fig. 6) it is located in the van der Veen \& Habing class VIII-area. 
The brightness of the variable is not compatible with the infrared fluxes. The inspection of the ESO/SRC Atlas revealed two red objects about 1.'0 and 1'.5 to the south of the variable, which may be the counterpart of the IRAS source.

$A U$ Circini: The infrared emission increases $\propto \lambda^{2}$, but the data for the $12 \mu \mathrm{m}$ flux are of poor quality and at $25 \mu \mathrm{m}$ there is an upper limit only. Nevertheless, the SED points to the presence of cold dust.

The weakness of the IRAS fluxes led us to expect a very weak optical counterpart. The eclipsing binary is, however, rather bright $\left(10^{\mathrm{m}} 7\right)$. Therefore, it is very unlikely to be the counterpart of the infrared source. The inspection of the ESO/SRC Atlas revealed no convincing candidate. There may be a red object between the merged images of two closely neighboured stars $\sim 1^{\prime}$ south of the variable. A second possible counterpart is situated $\sim 1^{\prime}$ west of that double star.

AT Normae: The IRAS fluxes at 12,25 , and $60 \mu \mathrm{m}$ are compatible with emission from warm dust. The 100$\mu \mathrm{m}$ flux, however, is by a factor of 50 higher than expected. This infrared emission can be explained by very cold dust. According to its position in the colour-colour diagram (Fig. 6), the IRAS source belongs to the van der Veen \& Habing classes VIa or VII, indicating a red evolved star.

Inspection of the ESO/SRC Atlas showed that the weak eclipsing variable itself is not red. A brighter star coinciding with the IRAS position seems to be a more promising candidate. However, this star does not appear red. Two weaker red stars which are additional possible candidates are also marked on the map.

$V 718$ Scorpii: The infrared radiation peaks at $25 \mu \mathrm{m}$ which points to warm circumstellar dust. The identification of V 718 Sco with IRAS 16102-2221 has been suggested by several authors (e.g., Gregorio-Hetem et al. 1992; Oudmaijer et al. 1992; Carballo et al. 1992). The true nature of the binary is not yet clear, but it may be a Herbig $\mathrm{Ae} / \mathrm{Be}$ object rather than a post-AGB object (van der Veen et al. 1994). The infrared source is close to location of disks after Smith et al. (1990).

AF Arae: The IRAS fluxes vary $\propto \lambda^{-1}$. The spectral type of the eclipsing variable is not known. There is no plausible value of the effective temperature that makes the visual magnitude of the variable compatible with the IRAS data.

The IRAS source belongs to the van der Veen \& Habing class VII and is expected to be a very red star. From the inspection of the ESO/SRC Atlas we conclude that the eclipsing binary is not red. A red object is found $\sim 1^{\prime}$ south to the variable, and we suggest it to be the counterpart of the IRAS source.

$V 463$ Scorpii: The infrared source belongs to our Group A and the van der Veen \& Habing class VII. The IRAS low resolution spectrum of this source (Fig. 8) shows an emission peak at $11.2 \mu \mathrm{m}$, which is attributed to circumstellar SiC grains. The infrared source should, therefore, be associated with a C-rich star surrounded by an optically thin dust envelope (Little-Marenin et al. 1987).

The inspection of the POSS prints revealed a red object $\sim 100^{\prime \prime}$ east of the IRAS position. Although this separation is rather large, the large magnitude makes this star a promising candidate for the infrared source. Two other red stars are very close to the IRAS position and deserve consideration.

$V 499$ Scorpii: The IRAS data indicate that the SED peaks at $60 \mu \mathrm{m}$ or beyond. Hence, the source belongs to our group III. No NIR data are known.

The eclipsing binary has a spectral type of B1V. If the IR source is identical with its circumstellar shell, the dust must be located well outside the binary system at a distance of about $0.05 \mathrm{pc}$.

An alternative explanation of the infrared radiation could be a very red object with an optically thick dust shell. An inspection of the POSS prints revealed a red star in the immediate vicinity of the eclipsing variable.

$B S$ Scorpii: The IRAS data indicate the presence of warm circumstellar dust. Neither NIR nor LRS data are available.

The binary has a spectral type of B5 V (Halbedel 1984). Neglecting interstellar extinction, we derive a photometric distance of $3.8 \mathrm{kpc}$. Allowing for an interstellar extinction of 2 mag (Neckel \& Klare 1980), the distance is more likely around $1.5 \mathrm{kpc}$.

Unfortunately, an HII region in the vicinity of the variable prevented the search for other possible counterparts of the IRAS source. The infrared source is close to the location of disks in Fig. 4 after Smith et al. (1990).

$V$ 792 Sagittarii: According to the IRAS data the infrared radiation increases $\propto \lambda^{2}$ and peaks beyond $100 \mu \mathrm{m}$. No NIR measurements are listed in the Catalog of Infrared Observations (Gezari et al. 1993). Tapia (1981) listed no infrared source at or near the IRAS position. The location of the source in the colour-colour diagram (Fig. 6) is compatible with the van der Veen \& Habing class VIII, which contains different types of infrared sources. A comparison with model computations by Gürtler et al. (1991) suggests that an optically very thick dust envelope $\left(A_{V} \approx 100 \mathrm{mag}\right)$ is compatible with the observations. The extrapolation of the SED into the visual region indicates a brightness of about $18^{\mathrm{m}}$, i.e. much fainter than the brightness of the variable. We conclude, therefore, that the eclipsing binary is not the optical counterpart of the infrared source.

Inspection of the POSS prints revealed a red star southeast of the variable. We suggest it as a more likely counterpart of the IRAS source than V 792 Sgr.

W Scuti: The SED of W Sct is similar to that of VV Ori. It peaks at $60 \mu \mathrm{m}$ or beyond and indicates the emission from cold dust. The components of the eclipsing binary are of spectral type B0 and B3. The photometric 
data suggest an interstellar extinction $A_{V} \approx 3$ mag. Nevertheless, the infrared excess is quite large, so that doubts about this identification remain. Inspection of the POSS prints shows two red objects at a distance of about $1^{\prime}$ east and south of the variable.

$R Y$ Scuti: The SED in the infrared is rather flat. The binary belongs to the group of the W Ser stars, which are contact binaries of early spectral type with large mass exchange rates (Aslanov et al. 1989). Even if the large interstellar extinction of $A_{V}=4.3 \mathrm{mag}$ is taken into account, the $12-\mu \mathrm{m}$ flux is largely due to emission from the circumstellar matter.

$\beta$ Lyrae: The SED varies $\propto \lambda^{-1}$ and indicates the presence of circumstellar matter. $\beta$ Lyr is a member of the $\mathrm{W}$ Ser group of eclipsing binaries.

$T Y$ Coronae Australis: The infrared emission is peaking beyond $100 \mu \mathrm{m}$, pointing to very cold dust. The binary is situated near the reflection nebula NGC 6726-7 and classified as a Herbig Ae/Be star. Bibo et al. (1992) concluded that the infrared emission comes from heated dust in the surrounding molecular cloud rather than from a circumstellar shell.

$V$ 1062 Sagittarii: The IRAS data show that the radiation peaks below $12 \mu \mathrm{m}$. The SED resembles that of SY Cru and points to a cool star with IR excess. There are no NIR data available. Te Lintel Hekkert et al. (1991) searched the IRAS source unsuccessfully for $1912 \mathrm{MHz}$ maser emission.

The spectral type of the binary is unknown. Inspection of the POSS prints shows that there is a very red object about $1^{\prime}$ west of the variable. This distance is comparable with the difference between the positions of the IRAS source and the binary. We conclude, therefore, that the red star is most probably the optical counterpart of the IRAS source.

$v$ Sagittarii: The IRAS fluxes varies $\propto \lambda^{-2}$. The LRS spectrum shows the $10-\mu \mathrm{m}$ silicate band in emission, indicating the presence of circumstellar dust. The comparison with the visual magnitude reveals a large infrared excess at $12 \mu \mathrm{m}$ and beyond. The properties of the infrared source and the binary speaks in favour of a post-AGB object (Trams et al. 1991).

HP Lyrae: The SED in the infrared agrees with the assumption of an origin from warm circumstellar dust. From the position of the infrared source in the colourcolour diagram (see Fig. 4) it belongs to the van der Veen \& Habing class IIIa. Typical members of this class are variables with evolved O-rich envelopes.

The eclipsing variable has the spectral type A6. This fact contradicts the classification by means of the IRAS data. Therefore, there are doubts about the identification of HP Lyr with the IRAS source in spite of the very good positional coincidence.
Inspection of the POSS prints revealed a red star in the immediate vicinity of HP Lyr, which we suggest as the counterpart of the IRAS source.

$\sigma$ Aquilae: The identity of the binary with the IRAS source was also noted by Gregorio-Hetem et al. (1992). The $12-\mu \mathrm{m}$ flux is only somewhat higher than expected from visual magnitude and spectral type. The SED as inferred from the IRAS fluxes peaks probably at $100 \mu \mathrm{m}$ or beyond and points to cool dust. The object is very similar to VV Ori.

EV Vulpeculae: The IRAS data show a steep increase of the infrared emission up to $100 \mu \mathrm{m}$ and beyond. The object belongs to our group C and is similar to V 792 Sgr. Because the infrared fluxes are about 20 times smaller than those of V 792 Sgr, we expect the optical counterpart to be fainter by about the same factor (i.e. by $\approx 3 \mathrm{mag}$ ) and thus to be a red star of $\approx 20^{\mathrm{m}}$. Inspection of the POSS prints revealed two red objects in the vicinity of the eclipsing variable.

V 501 Cygni: The IRAS fluxes indicate an envelope of cool dust. NIR data are not available. The IRAS data can be interpreted by a superposition of stellar radiation and thermalised dust emission. The transition from stellar to circumstellar emission is between 12 and $25 \mu \mathrm{m}$.

Inspection of the POSS prints shows a red object about $1^{\prime}$ south of the variable. This distance is comparable with the difference between the IRAS and star positions. Therefore, we conclude that the IRAS source is identical with the red star and not with the eclipsing binary.

$P S$ Cephei: The infrared source is located close to the edge of the Hil region Sh 142. The infrared emission varies $\propto \lambda^{2}$ between 12 and $100 \mu \mathrm{m}$, pointing to the emission by cool dust. The association of IRAS $22457+5751$ with PS Cep (= BD +572615$)$ was also noted by Dong \& Hu (1991). If PS Cep is actually associated with the infrared source, the case may be similar to TY CrA. Inspection of the ESO/SRC Atlas revealed a very red object to the east of the IRAS position. Thus, it could be that the counterpart of IRAS $22457+5751$ is a deeply embedded young object.

In addition to the stars of our "restricted sample" we have searched the rest of the stars for which the spectral type is known for obvious agreement between the optical brightness and the $12-\mu \mathrm{m}$ flux. In Table 3 we collected all eclipsing binary stars for which visual magnitude and $12-\mu \mathrm{m}$ flux are compatible with each other and which, therefore, we consider reliable associations. As far as the $25-\mu \mathrm{m}$ flux is also known, the ratio of the $12-\mu \mathrm{m}$ to $25-\mu \mathrm{m}$ fluxes points to a stellar rather than circumstellar origin of the radiation in almost all cases.

In Table 4 we present an additional subsample of eclipsing binaries that are associated with IRAS point sources. These sources have separations $D<15^{\prime \prime}$ and were not included in our "restricted sample" because there are upper limits for the fluxes at 60 and $100 \mu \mathrm{m}$. Finding 
Table 3. List of eclipsing stars certainly associated with IRAS point sources

\begin{tabular}{|c|c|c|c|c|c|c|c|c|}
\hline \multirow[t]{2}{*}{ Variable star } & \multirow[t]{2}{*}{ Spectral type } & \multirow[t]{2}{*}{ IRAS source } & \multirow{2}{*}{$\begin{array}{c}\text { Separation } \\
\left({ }^{\prime \prime}\right) \\
\end{array}$} & \multicolumn{4}{|c|}{ Infrared fluxes (Jy) } & \multirow[t]{2}{*}{ Remarks } \\
\hline & & & & $12 \mu \mathrm{m}$ & $25 \mu \mathrm{m}$ & $60 \mu \mathrm{m}$ & $100 \mu \mathrm{m}$ & \\
\hline YZ Cas & $\mathrm{A} 2 \mathrm{~V}+\mathrm{F} 2 \mathrm{~V}$ & $00423+7443$ & 14 & 0.24 & $<0.25$ & $<0.40$ & $<1.78$ & \\
\hline CF Tuc & $\mathrm{F} 8 \mathrm{~V}+\mathrm{K} 1 \mathrm{IV}$ & $00515-7455$ & 13 & 0.36 & $<0.25$ & $<0.40$ & $<1.03$ & \\
\hline RR Ari & K0III & $01530+2319$ & 6 & 2.79 & 0.62 & $<0.40$ & $<1.34$ & \\
\hline RZ Cas & $\mathrm{K} 1 \mathrm{III}+\mathrm{A} 5 \mathrm{eIII}$ & $03032+6723$ & 7 & 0.41 & $<0.25$ & $<0.40$ & $<12.93$ & \\
\hline$\beta$ Per & $\mathrm{B} 8 \mathrm{~V}$ & $03048+4015$ & 11 & 7.36 & 1.89 & $<0.52$ & $<1.37$ & \\
\hline TZ For & G0III+ G0III & $03126-3544$ & 3 & 0.46 & $<0.25$ & $<0.40$ & $<1.00$ & \\
\hline V 467 Per & $\mathrm{A} 2 \mathrm{~V}$ & $03463+3256$ & 5 & 0.50 & $<0.28$ & $<0.40$ & $<12.34$ & \\
\hline$\lambda \mathrm{Tau}$ & $\mathrm{B} 3 \mathrm{~V}+\mathrm{A} 4 \mathrm{IV}$ & $03579+1221$ & 4 & 1.30 & $0.31:$ & $<0.40$ & $<4.46$ & \\
\hline$\vartheta^{2} \mathrm{Tau}$ & A7III & $04257+1545$ & 24 & 3.02 & 0.69 & $<0.40$ & $<2.01$ & \\
\hline$\eta$ Ori & $\mathrm{B} 0.5 \mathrm{Vea}+\mathrm{B} 3 \mathrm{~V}$ & $05219-0226$ & 2 & 1.05 & $<0.27$ & $<0.40$ & $<8.82$ & \\
\hline$\psi$ Ori & B2IVea+B0 & $05241+0303$ & 31 & 0.29 & $<0.25$ & $<0.40$ & $<1.28$ & \\
\hline$\delta$ Ori & O9.5II-III+B0III & 05294-0020 & 2 & 3.05 & 0.84 & $<5.32$ & $<971.07$ & \\
\hline TU Cam & A0IV-V & $05504+5952$ & 8 & 0.32 & $<0.25$ & $<0.40$ & $<1.14$ & \\
\hline$\beta$ Aur & $\mathrm{A} 2 \mathrm{IV}+\mathrm{A} 2 \mathrm{IV}-\mathrm{V}$ & $05558+4456$ & 11 & 7.85 & 1.63 & $<0.40$ & $<1.00$ & \\
\hline$\delta \mathrm{Pic}$ & B3III+O9V & $06092-5457$ & 39 & 0.26 & $<0.25$ & $<0.47$ & $<1.24$ & \\
\hline UZ Lyn & $\mathrm{A} 2 \mathrm{~V}$ & $06151+5901$ & 9 & 0.73 & $0.23:$ & $<0.40$ & $<1.00$ & \\
\hline RR Lyn & $\mathrm{A} 7 \mathrm{Vm}+\mathrm{F} 3 \mathrm{~V}$ & $06222+5618$ & 5 & 0.41 & $<0.38$ & $<0.40$ & $<1.00$ & \\
\hline UW CMa & O7Ia:fp+OB & $07165-2427$ & 3 & 0.37 & $<0.25$ & $<0.43$ & $<12.76$ & \\
\hline $\mathrm{R} \mathrm{CMa}$ & F1V & $07172-1618$ & 2 & 0.65 & $<0.27$ & $<0.43$ & $<12.69$ & \\
\hline PU Pup & B9 & $07362-2514$ & 2 & 0.48 & $<0.25$ & $<0.40$ & $<10.65$ & \\
\hline UU Cnc & K4III & $07597+1519$ & 53 & 0.32 & $<0.29$ & $<0.40$ & $<1.16$ & \\
\hline NO Pup & $\mathrm{B} 8 \mathrm{~V}$ & $08244-3853$ & 23 & 0.27 & $<0.25$ & $<0.47$ & $<24.30$ & \\
\hline RS Cha & $\mathrm{A} 5 \mathrm{~V}+\mathrm{A} 7 \mathrm{~V}$ & $08450-7854$ & 65 & $<0.35$ & 0.31 & $<0.54$ & $<1.45$ & \\
\hline TY Pyx & $\mathrm{G} 5+\mathrm{G} 5$ & $08575-2737$ & 23 & 0.37 & $<0.27$ & $<0.40$ & $<1.16$ & \\
\hline V 257 Car & B2IV-V & $09096-5845$ & 6 & 1.00 & 0.22 & $<0.43$ & $<1.83$ & \\
\hline KW Hya & A3III+A0V: & 09099-0654 & 15 & 0.25 & $<0.25$ & $<0.40$ & $<1.00$ & \\
\hline IP Vel & $\mathrm{B} 6 \mathrm{~V}$ & $09417-5058$ & 69 & 0.41 & $<0.25$ & $<0.40$ & $<11.53$ & \\
\hline V 788 Cen & $\mathrm{A} 2 \mathrm{~mA} 5-\mathrm{F} 2$ & $12062-4402$ & 2 & 0.36 & $<0.25$ & $<0.42$ & $<1.52$ & \\
\hline$\vartheta$ Mus & $\mathrm{WC} 6+09.5 \mathrm{I}$ & $13048-6502$ & 3 & 0.47 & $<0.25$ & $<1.74$ & $<15.96$ & \\
\hline$\eta \mathrm{Mus}$ & $\mathrm{B} 8 \mathrm{~V}$ & $13117-6737$ & 39 & 0.35 & $<0.25$ & $<0.68$ & $<6.80$ & \\
\hline AO Mus & $\mathrm{C}(\mathrm{R})$ & $13378-6733$ & 11 & 0.26 & $<0.24$ & $<0.49$ & $<4.63$ & \\
\hline$\delta \mathrm{Lib}$ & A0IV-V & $14582-0819$ & 1 & 0.68 & $<0.47$ & $<0.40$ & $<1.00$ & \\
\hline V 760 Sco & $\mathrm{B} 3: \mathrm{Vn}$ & $16214-3448$ & 89 & $<0.27$ & $<0.51$ & 1.74 & $<15.02$ & \\
\hline$\mu^{1}$ Sco & $\mathrm{B} 1.5 \mathrm{~V}+\mathrm{B} 6.5 \mathrm{~V}$ & $16484-3758$ & 40 & 2.17 & 1.04 & $<1.04$ & $<12.01$ & \\
\hline i Boo & $\mathrm{G} 2 \mathrm{~V}+\mathrm{G} 2 \mathrm{~V}$ & $15021+4750$ & 1 & 2.38 & 0.60 & $<0.40$ & $<1.00$ & \\
\hline$\alpha \mathrm{CrB}$ & $\mathrm{A} 0 \mathrm{~V}+\mathrm{G} 5 \mathrm{~V}$ & $15325+2652$ & 6 & 5.92 & 1.73 & $<0.75$ & $<1.00$ & \\
\hline u Her & $\mathrm{B} 1.5 \mathrm{Vp}+\mathrm{B} 5 \mathrm{III}$ & $17154+3309$ & 1 & 0.34 & $<0.25$ & $<0.40$ & $<1.14$ & \\
\hline$\lambda \mathrm{Sco}$ & B1.5IV & $17302-3704$ & 5 & 4.43 & 1.19 & $<5.08$ & $<129.58$ & \\
\hline V 772 Her & $\mathrm{G} 2 \mathrm{~V}$ & $18036+2126$ & 24 & 0.39 & $<0.25$ & $<0.40$ & $<1.10$ & \\
\hline$\mu \mathrm{Sgr}$ & $\mathrm{B} 8 \mathrm{eqIa}+\mathrm{B} 1.5 \mathrm{~V}$ & $18107-2104$ & 1 & 3.09 & 0.99 & $<16.45$ & $<256.28$ & \\
\hline$\epsilon \mathrm{CrA}$ & $\mathrm{F} 2 \mathrm{~V}$ & $18553-3710$ & 5 & 1.26 & $<0.52$ & $<0.41$ & $<6.24$ & \\
\hline BL Tel & $\mathrm{F} 5 \mathrm{Iab} / \mathrm{b}+\mathrm{M}$ & $19027-5129$ & 4 & 0.61 & $<0.25$ & $<0.40$ & $<1.01$ & \\
\hline Z Vul & $\mathrm{B} 4 \mathrm{~V}+\mathrm{A} 3 \mathrm{III}$ & $19196+2529$ & 23 & $<0.28$ & $<0.26$ & 0.83 & $<2.26$ & \\
\hline V 1143 Cyg & $\mathrm{F} 5 \mathrm{~V}+\mathrm{F} 5 \mathrm{~V}$ & $19375+5451$ & 1 & 0.50 & $<0.25$ & $<0.40$ & $<1.28$ & \\
\hline CI Cyg & Bep+M5III & $19483+3533$ & 9 & 0.70 & 0.28 & $<0.45$ & $<5.48$ & \\
\hline V 2235 Sgr & $\mathrm{Mb}$ & $20041-3640$ & 24 & 4.26 & 1.25 & $<0.40$ & $<1.00$ & \\
\hline V 695 Cyg & $\mathrm{K} 4 \mathrm{Iab}+\mathrm{B} 4 \mathrm{IV}-\mathrm{V}$ & $20120+4635$ & 3 & 28.84 & 8.04 & $<1.98$ & $<14.32$ & LRS \\
\hline V 1488 Cyg & $\mathrm{K} 5 \mathrm{Iab}+\mathrm{B} 4 \mathrm{IV}-\mathrm{V}$ & $20139+4733$ & 6 & 42.52 & 10.32 & $<1.25$ & $<53.22$ & LRS \\
\hline VW Cep & G5+K0ve & $20381+7525$ & 0 & 0.41 & $<0.25$ & $<0.40$ & $<2.04$ & \\
\hline$\delta$ Cap & $\mathrm{A} 7+\mathrm{MIII}$ & $21442-1621$ & 5 & 6.24 & 1.50 & $<0.40$ & $<1.00$ & \\
\hline DX Aqr & $\mathrm{A} 2 \mathrm{~V}$ & $21597-1712$ & 8 & 0.57 & $<0.33$ & $<0.40$ & $<1.00$ & \\
\hline AR Lac & $\mathrm{G} 2 \mathrm{IV}+\mathrm{K} 0 \mathrm{IV}$ & $22066+4529$ & 16 & 0.85 & $<0.25$ & $<0.40$ & $<3.33$ & \\
\hline AW Gru & M4III & $22160-4817$ & 15 & 2.68 & 0.71 & $<0.40$ & $<1.00$ & \\
\hline SZ Psc & $\mathrm{K} 1 \mathrm{IV}-\mathrm{V}+\mathrm{F} 8 \mathrm{~V}$ & $23108+0224$ & 18 & 0.50 & $<0.26$ & $<0.40$ & $<1.01$ & \\
\hline V 373 Cas & $\mathrm{B} 0.5 \mathrm{II}+\mathrm{B} 0.5 \mathrm{II}$ & $23530+5707$ & 5 & 0.30 & $<0.34$ & $<0.72$ & $<0.72$ & \\
\hline
\end{tabular}


Table 4. List of additional eclipsing stars closely associated with IRAS point sources

\begin{tabular}{llcrrrrr}
\hline Variable star & Spectral type & IRAS point source & $\begin{array}{c}\text { Separation } \\
\left({ }^{\prime}\right)\end{array}$ & \multicolumn{4}{c}{ Infrared fluxes (Jy) } \\
& & & $12 \mu \mathrm{m}$ & $25 \mu \mathrm{m}$ & $60 \mu \mathrm{m}$ & $100 \mu \mathrm{m}$ \\
\hline FY Vel & B2Ib pe & $08308-4925$ & 11 & 1.17 & 0.80 & $<0.58$ & $<16.39$ \\
HX Cen & & $13517-5132$ & 12 & 0.53 & $0.26:$ & $<0.40$ & $<2.19$ \\
IV Ara & & $17265-5809$ & 11 & 2.26 & 1.38 & $<0.40$ & $<3.69$ \\
V 924 Oph & & $18310+0755$ & 10 & 0.52 & 0.34 & $<0.40$ & $<2.48$ \\
FR Tel & Me & $18378-5154$ & 15 & 0.77 & 0.46 & $<0.40$ & $<1.11$ \\
V 929 Oph & & $18385+0814$ & 2 & 1.35 & 0.76 & $<0.40$ & $<3.34$ \\
PS Vul & B7V + G1:III & $19418+2700$ & 6 & 3.86 & 0.94 & $<0.83$ & $<42.98$ \\
AT Vul & B3 & $19518+2326$ & 4 & $<0.25$ & 0.80 & 7.09 & $<13.26$ \\
V 484 Cyg & & $19588+3506$ & 13 & 2.19 & 0.95 & $<4.49$ & $<71.56$ \\
V 729 Cyg & O7f Ia + O8 & $20305+4108$ & 6 & 1.47 & 1.11 & $<12.08$ & $<119.74$ \\
V 367 Cyg & B8peIa + F4III & $20460+3906$ & 5 & 1.13 & 0.57 & $<0.65$ & $<4.14$ \\
IL Cep & B2-B3pe & $22513+6152$ & 7 & 2.70 & 2.30 & $<5.21$ & $<49.19$ \\
\hline
\end{tabular}

charts are given in Fig. 9. The following detailed discussion of these binaries will show that most of them seem to really associated with the IRAS sources in accordance with the expectations from Fig. 2.

FY Velorum: The association of this star with IRAS 08308-4925 was also noted by Oudmaijer et al. (1992). The binary is classified as a member of the W Ser stars. After correcting its visual magnitude for interstellar extinction, the ratio of $12-\mu \mathrm{m}$ flux to visual brightness is comparable to that of $\beta$ Lyr, another member of this group.

$H X$ Centauri: Little information is available about this star. It appears not red on the ESO/SRC Atlas. Inspection of that atlas revealed two red objects. The fainter is within the error box of the IRAS source. However, the object north to the IRAS position seems to be a better candidate because of its larger brightness.

IV Arae: No spectral type is known of this binary. Inspection of the ESO/SRC Atlas revealed three red objects as possible candidates of the IRAS source. The southeastern star with a magnitude of about $13{ }^{\mathrm{m}} 6$ is the most promising candidate.

$V 924$ Ophiuchi: Inspection of the POSS prints showed that the variable appears red. Its visual brightness is estimated at $\approx 14^{\mathrm{m}} 5$. Such a magnitude is compatible with the $12-\mu \mathrm{m}$ flux measured by IRAS. Therefore, we conclude that V 924 Oph and IRAS $18310+0755$ are very probably identical.

FR Telescopii: Considering the spectral type Me as given in the GCVS, the $12-\mu \mathrm{m}$ flux matches the optical brightness of the star. So we feel that the binary and the IRAS source are identical.

$V$ 929 Ophiuchi: The variable appears very red on the POSS prints. Therefore, we suggest that the binary and the IRAS source are identical. No spectrum of the binary is known. The IRAS observations are compatible with the emission of circumstellar dust. As was pointed out by Friedemann \& Löwe (1990) the infrared source is close to the location of disks in Fig. 4 after Smith et al. (1990).

$P S$ Vulpeculae: The colour index of $B-V=1.10 \mathrm{mag}$ indicates large extinction. If this is taken into account, the visual magnitude is fully compatible with the $12-\mu \mathrm{m}$ flux.

AT Vulpeculae: The IRAS observations suggest that the infrared source is similar to V $792 \mathrm{Sgr}$ or EV Vul. From the photometry of Lacy (1992) we estimated an interstellar extinction of $A_{V} \approx 2 \mathrm{mag}$. The visual magnitude of the binary is then compatible with the expectations from the IRAS observations.

$V 484$ Cygni: The $12-\mu \mathrm{m}$ flux is rather large for the visual brightness of the binary, which does not appear red on the POSS prints. Inspection of the POSS reveals several red objects. We have indicated the brightest only, which we feel is a more probable candidate than the binary.

$V 729$ Cygni: If the apparent visual magnitude is corrected for the large interstellar extinction $\left(A_{V}=6.40\right.$ mag; Torres-Dodgen et al. 1991), the visual brightness of the star is fully compatible with the $12-\mu \mathrm{m}$ flux. The flatness of the infrared spectrum as indicated by the $25-\mu \mathrm{m}$ flux may point to free-free emission as the origin of the far-infrared radiation.

V 367 Cygni: This binary belongs to the W Ser stars. If a plausible amount of interstellar extinction is allowed for, the ratio of visual magnitude to infrared flux is for V 387 Cyg the same as that for $\beta$ Lyr, another member of this group.

IL Cephei: This binary belongs to the group of the Herbig Ae/Be stars. Its association with IRAS 22513+6152 was previously noted by Dong \& Hu (1991) and Oudmaijer et al. (1992). Photometric observations by Blaauw et al. (1959) imply an interstellar or circumstellar extinction of $A_{V}=3$ mag. After correction for this amount of extinction, the position in the $S(12)-V$ diagram (Fig. 3) suggests that the $12-\mu \mathrm{m}$ flux observed by IRAS is about 10 times the flux expected from the stellar photosphere. 


\section{Conclusions}

1. We performed a systematic search for counterparts of IRAS point sources among eclipsing binaries by means of positional coincidences. Altogether, 233 positional associations better than $90^{\prime \prime}$ were found.

2. The IRAS photometric data show the infrared sources to have a wide variety of spectral energy distributions indicating that quite different infrared sources are involved.

3 . The statistical analysis and comparison with model calculations make it very probable that $50-75 \%$ of the eclipsing binaries are associated with IRAS point sources.

4. Among the brighter eclipsing binaries virtually all are physically associated with IRAS sources. However, in many cases the existing infrared data are too scarce to allow conclusions on the origin of the infrared radiation.

5. A detailed discussion of a subsample of 44 sources for which the flux was measured in at least three wavelength bands by IRAS showed that 20 eclipsing binaries are probably associated with IRAS point sources.

6. From a search of the POSS, SRC and ESO atlases, we report possible optical counterparts of the IRAS point sources.

7. In the cases of BS Sco and V 718 Sco the position of the star in the IRAS colour-colour diagram is compatible with the emission coming from a circumstellar disk.

Acknowledgements. The authors are indebted to Drs. Th. Neckel, MPIA Heidelberg, and O. Fischer, UniversitätsSternwarte Jena, for providing them with some copies of the ESO and SRC sky surveys. They wish to thank Dr. E. Krügel, MPIfR Bonn, for making his radiative transfer programme available to them. The paper has profited much by comments by Dr. W. Pfau, Universitäts-Sternwarte Jena, and the referee, Dr. M. Grewing. This work made use of the SIMBAD data base, operated at CDS, Strasbourg, France. This work was partly supported by the Deutsche Forschungsgemeinschaft (Fr 963-1) and by the German Bundesministerium für Bildung, Wissenschaft, Forschung und Technologie (Förderkennzeichen 05 2JN13A).

\section{References}

Arp H.C., van Sant C.T., 1958, AJ 63, 341
Aslanov A.A., Kolosov D. Ye., Lipunova N.A., Khruzina T.S., Cherepashchuk A.M., 1989, Katalog tesnykh dvoinykh zvezd na pozdnikh stadiyakh Evolyutsii. Moscow University Press, Moscow

Bibo E.A., Thé P.S., Dawanas D.N., 1992, A\&A 260, 293

Blaauw A., Hiltner W.A., Johnson H.L., 1959, ApJ 130, 69

Caldwell J.A.R., Keane M.J., Schechter P.L., 1991, AJ 101, 1763

Carballo R., Wesselius P.R., Whittet D.C.B, 1992, A\&A 262, 106

Dong Y.S., Hu J.Y., 1991, Acta Astrophys. Sinica 11, 172

Feast M.W., 1963, MNRAS 126, 11

Friedemann C., Löwe M., 1990, IAU Inform. Bull. Var. Stars, No. 3516

Gezari D.Y, Schmitz M., Pitts P.S., Mead J.M., 1993, Catalog of Infrared Observations. NASA Reference Publication RP1294

Gregorio-Hetem J., Lépine J.R.D., Quast G.R., Torres C.A.O., de la Reza R., 1992, AJ 103, 549

Gürtler J., Henning Th., Krügel E., Chini R., 1991, A\&A 252, 801

Halbedel E.M., 1984, IAU Inform. Bull. Var. Stars, No. 2549

Herczeg T., 1982, in Landolt-Börnstein, Zahlenwerte und Funktionen aus Naturwissenschaft und Technik, NS, Gr. VI, Vol. 2b, p. 381

Joint IRAS Science Working Group, 1988, IRAS Point Source Catalogue, NASA, Washington

Kholopov P.N., 1985 General Catalogue of Variable Stars, 4th ed., Nauka, Moscow

Lacy C.H., 1992, AJ 104, 801

Little-Marenin I., Ramsay M.E., Stephenson C.B., Little S.J., Price S.D., 1987, AJ 93, 663

Neckel Th., Klare G., 1980, A\&AS 42, 251

Oudmaijer R.D., van der Veen W.E.C.J., Waters L.B.F.M., Trams N.R., Waelkens C., Engelsman E., 1992, A\&AS 96 , 625

Smith H.A., Beall J.H., Swain M.R., 1990, AJ 99, 273

Tapia M., 1981, MNRAS 197, 949

te Lintel-Hekkert P., Caswell J.L., Habing H.J., Haynes R.F., Norris R.P., 1991, A\&A 90, 327

Torres-Dodgen A.V., Tapia M., Carrol M., 1991, MNRAS 249, 1

Trams N.R., Waters L.B.F.M., Lamers H.J.G.L., Waelkens C., Geballe T.R., Thé P.S., 1991, A\&AS 87, 361

van der Veen W.E.C.J., Habing H.J., 1988, A\&A 194, 125

van der Veen W.E.C.J., Waters L.B.F.M., Trams N.R., Matthews H.E., 1994, A\&A 285, 551

Walker, H.J., 1985, A\&A 152, 58

Walker H.J., Cohen M., Volk K., Wainscoat R.J., Schwartz D.E., 1989, AJ 98, 2163 